\title{
Type III TGF- $\beta$ receptor downregulation generates an immunotolerant tumor microenvironment
}

\author{
Brent A. Hanks, ${ }^{1}$ Alisha Holtzhausen, ${ }^{2}$ Katherine S. Evans, ${ }^{1}$ Rebekah Jamieson, ${ }^{2}$ Petra Gimpel, ${ }^{3}$ \\ Olivia M. Campbell, 2 Melissa Hector-Greene, ${ }^{2,4}$ Lihong Sun, ${ }^{5}$ Alok Tewari, ${ }^{4}$ Amanda George, ${ }^{1}$ \\ Mark Starr, ${ }^{1}$ Andrew B. Nixon, ${ }^{1}$ Christi Augustine, ${ }^{6}$ Georgia Beasley, ${ }^{6}$ Douglas S. Tyler, ${ }^{6}$ \\ Takayu Osada, ${ }^{6}$ Michael A. Morse, ${ }^{1}$ Leona Ling, ${ }^{5}$ H. Kim Lyerly, ${ }^{6}$ and Gerard C. Blobe ${ }^{1,2}$
}

1Department of Medicine and 'Department of Pharmacology and Cancer Biology, Duke University Medical Center, Durham, North Carolina, USA. ${ }^{3}$ Freie Universität Berlin, Institut für Chemie und Biochemie, Berlin, Germany. ${ }^{4}$ Medical Scientist Training Program, Duke University Medical Center, Durham, North Carolina, USA. ${ }^{5}$ Biogen Idec Inc., Cambridge, Massachusetts, USA. ${ }^{6}$ Department of Surgery,

Duke University Medical Center, Durham, North Carolina, USA.

\begin{abstract}
Cancers subvert the host immune system to facilitate disease progression. These evolved immunosuppressive mechanisms are also implicated in circumventing immunotherapeutic strategies. Emerging data indicate that local tumor-associated DC populations exhibit tolerogenic features by promoting Treg development; however, the mechanisms by which tumors manipulate DC and Treg function in the tumor microenvironment remain unclear. Type III TGF- $\beta$ receptor (TGFBR3) and its shed extracellular domain (sTGFBR3) regulate TGF- $\beta$ signaling and maintain epithelial homeostasis, with loss of TGFBR3 expression promoting progression early in breast cancer development. Using murine models of breast cancer and melanoma, we elucidated a tumor immunoevasion mechanism whereby loss of tumor-expressed TGFBR3/sTGFBR3 enhanced TGF- $\beta$ signaling within locoregional DC populations and upregulated both the immunoregulatory enzyme indoleamine 2,3-dioxygenase (IDO) in plasmacytoid DCs and the CCL22 chemokine in myeloid DCs. Alterations in these DC populations mediated Treg infiltration and the suppression of antitumor immunity. Our findings provide mechanistic support for using TGF- $\beta$ inhibitors to enhance the efficacy of tumor immunotherapy, indicate that sTGFBR3 levels could serve as a predictive immunotherapy biomarker, and expand the mechanisms by which TGFBR3 suppresses cancer progression to include effects on the tumor immune microenvironment.
\end{abstract}

\section{Introduction}

Tumor immunotherapy has demonstrated only modest clinical efficacy, largely due to the strategies used by tumors to evade the host immune system $(1,2)$. Many of these mechanisms create an immunotolerant microenvironment and suppress the generation of an effective antitumor immune response, allowing tumors to ultimately escape cancer immunosurveillance (3, 4).

A key component of the tumor immune microenvironment is the sentinel LN. As a result of soluble factors released by primary tumor tissues, APC populations within these locoregional LNs, including DCs, exhibit dampened immunostimulatory properties $(5,6)$. Although phenotypic alteration of APCs within the tumor microenvironment may be necessary for tumor progression (7), our understanding of both the tumor-derived factors targeting these cell populations and the specific modulatory effects that they elicit remains extremely limited.

TGF- $\beta$ is a potent immunosuppressive cytokine that inhibits $\mathrm{T}$ cell function and the antigen presentation capacity of DCs $(8,9)$. Recent studies have elucidated a direct role for TGF- $\beta$ in upregulating the expression of the immunoregulatory enzyme

Conflict of interest: The authors have declared that no conflict of interest exists.

Note regarding evaluation of this manuscript: Manuscripts authored by scientists associated with Duke University, The University of North Carolina at Chapel Hill, Duke-NUS, and the Sanford-Burnham Medical Research Institute are handled not by members of the editorial board but rather by the science editors, who consult with selected external editors and reviewers.

Citation for this article: J Clin Invest. 2013;123(9):3925-3940. doi:10.1172/JCI65745 indoleamine 2,3-dioxygenase (IDO) by plasmacytoid DCs (pDCs) and support a role for this pathway in long-term $\mathrm{T}$ cell tolerization $(10,11)$. By catalyzing the degradation of the essential amino acid tryptophan, IDO suppresses effector $\mathrm{T}$ cell activity and promotes Treg differentiation and activation (12-15). Interestingly, elevated tumor-draining LN (TDLN) IDO expression levels predict inferior clinical outcome in melanoma, which suggests that IDO is an important negative regulator of antitumor immunity (16, 17). In addition to activating Tregs via IDO, some tumors also use the DC-derived CCL22 chemokine to promote CCR4-dependent recruitment of these cells to the tumor microenvironment and to establish an immunoprotected sanctuary allowing for disease progression $(18,19)$. Despite this understanding, however, the mechanisms used by tumors to manipulate DC function to ultimately generate local immunosuppression remain unclear.

Type III TGF- $\beta$ receptor (TGFBR3) is a coreceptor for the canonical TGF- $\beta$ signaling pathway, with emerging roles in mediating both SMAD-dependent and SMAD-independent downstream signaling $(20,21)$. We have previously shown that TGFBR3 expression is downregulated during the early stages of progression of many human cancers, including breast cancer, compared with patientmatched normal tissue controls (22-24). Furthermore, we have shown that TGFBR3 inhibits cell migration and invasion in several tumor models, in support of a role for TGFBR3 as a suppressor of cancer progression and metastasis $(25,26)$. Mechanistically, TGFBR3 undergoes ectodomain shedding from the cell surface to generate a soluble form of TGFBR3 (sTGFBR3) that sequesters 


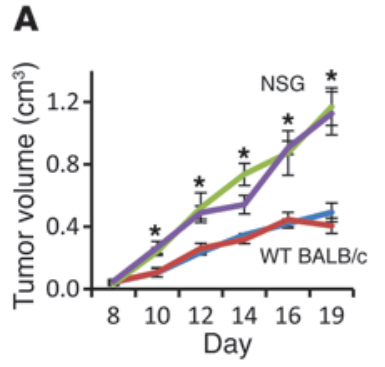

B
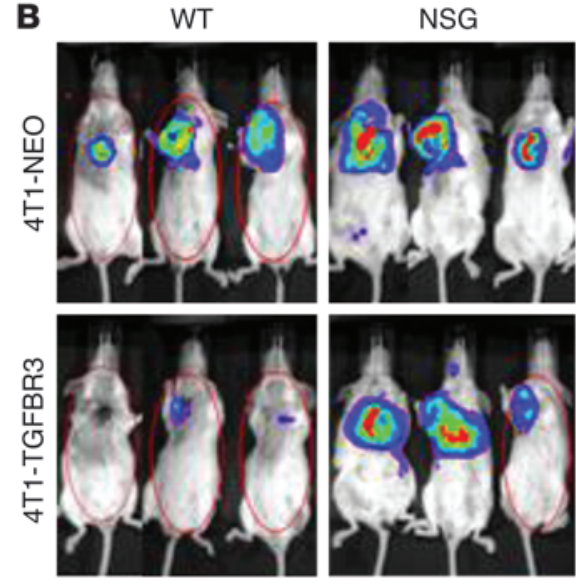

D
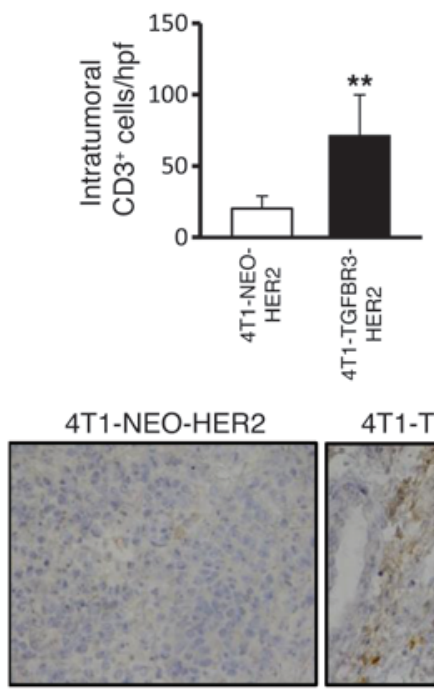

4T1-TGFBR3-HER2

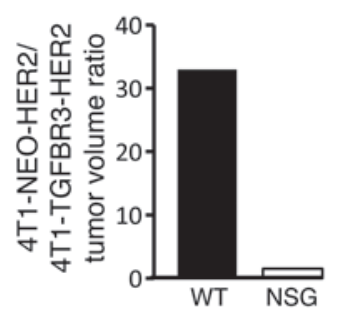

E

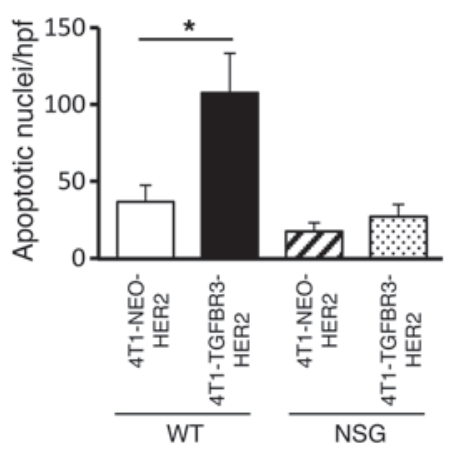

$\mathbf{F}$

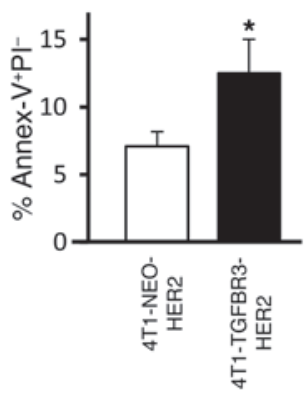

Figure 1

Loss of TGFBR3 expression promotes tumor progression only in immunocompetent hosts. (A) In vivo growth of $4 \mathrm{~T} 1-\mathrm{NEO}$ and 4T1-TGFBR3 primary tumors in NSG and WT hosts. 15 mice/ group. Red, 4T1-NEO WT; blue, 4T1-TGFBR3 WT; green, 4T1-NEO NSG; purple, 4T1-TGFBR3 NSG. (B) 4T1-NEO/4T1TGFBR3 tumor burden ratios, based on in vivo bioluminescence monitoring. Representative images are also shown. (C) 4T1-NEO-HER2 and 4T1-TGFBR3-HER2 tumor incidence and volume in NSG and WT hosts. $6 \mathrm{mice} / g r o u p$. Representative of 3 individual experiments. (D) CD3 IHC of 4T1-NEO-HER2 and 4T1-TGFBR3-HER2 tumors. 10 random $\times 40$ fields/condition (representative $\times 40$ images are also shown). Representative of 2 independent experiments. (E) TUNEL analysis of 4T1-NEOHER2 and 4T1-TGFBR3-HER2 tumors resected from WT and NSG hosts. 10 random $\times 40$ fields/ condition. Representative of 2 independent experiments. (F) Annexin-V/PI flow cytometry analysis of resected 4T1-NEO-HER2 and 4T1-TGFBR3-HER2 tumors from WT hosts. Data are mean \pm SEM. ${ }^{*} P<0.05,{ }^{* *} P<0.005$, 2-tailed Student's $t$ test.
TGF- $\beta$ superfamily ligands and inhibits their downstream signaling $(22,27,28)$. Here, we investigated the effects of tumor TGFBR3 downregulation on TGF- $\beta$ signaling within the immune microenvironment and its potential implications in cancer immunotherapy.

\section{Results}

Loss of TGFBR3 expression generates an immunotolerant tumor microenvironment. To determine whether TGFBR3 regulates antitumor immunity, we orthotopically implanted 4T1 murine mammary carcinoma cells expressing TGFBR3 (referred to herein as 4T1-TGFBR3 cells; Supplemental Table 1 and Supplemental Figure 1A; supplemental material available online with this article; doi:10.1172/ JCI65745DS1) and an empty vector control cell line (4T1-NEO cells) into the right mammary fat pad of either syngeneic female immunocompetent WT BALB/c mice or female immunocompromised IL-2 $\gamma$-chain-deficient NOD.Cg-Prkdc scidIl2rg $g^{t m 1 W j l} / \mathrm{Szj}$ 

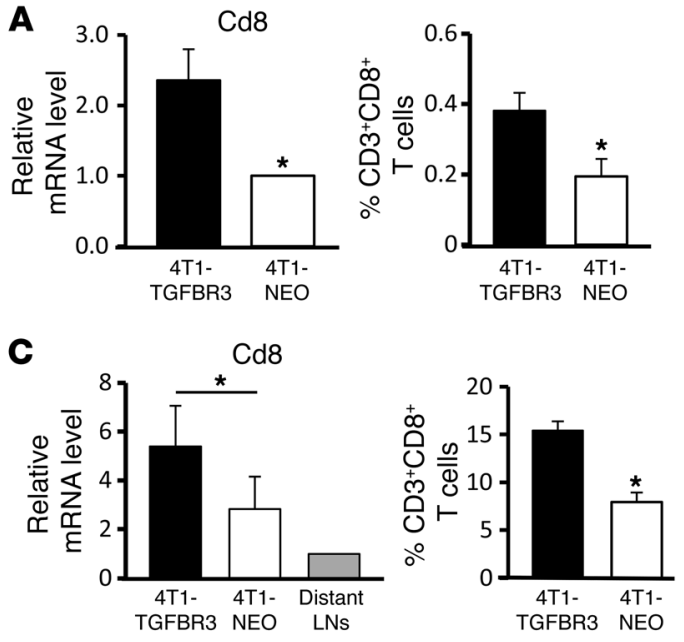

D
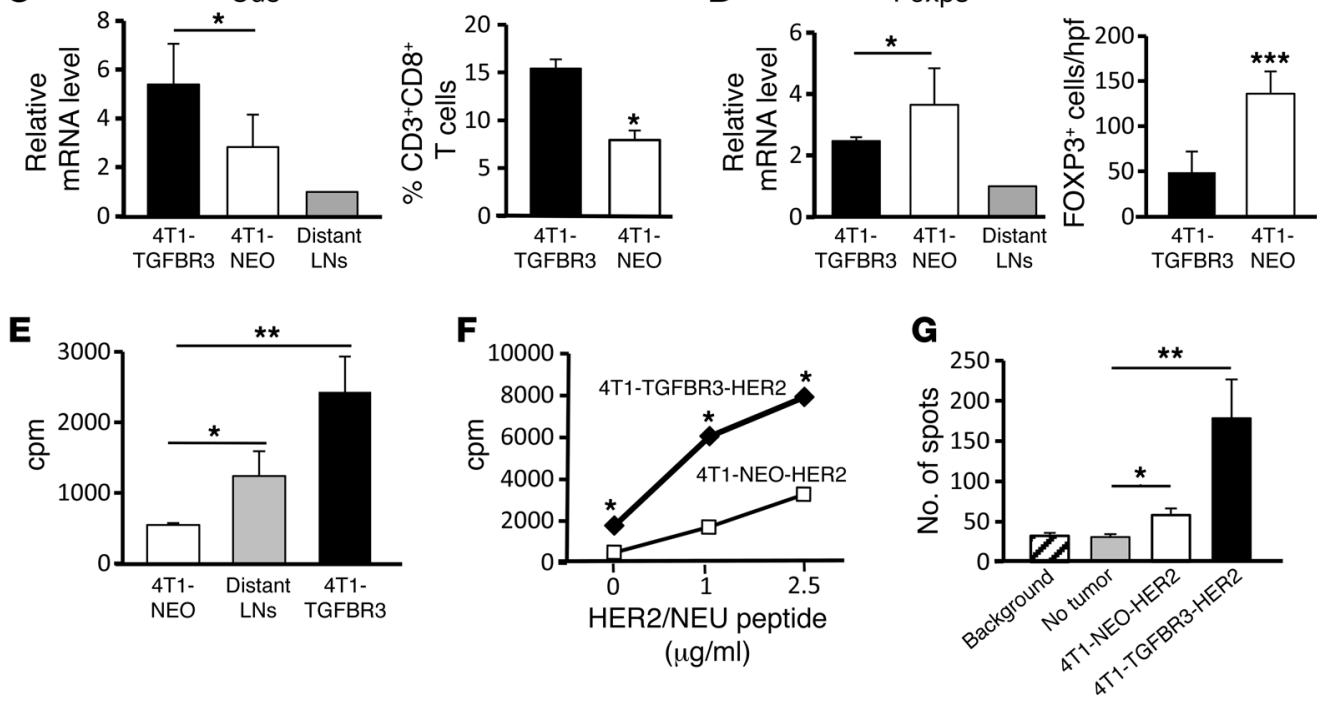

Figure 2

Loss of TGFBR3 expression generates an immunotolerant tumor microenvironment in the 4T1 murine breast cancer model. (A) Cd8 qRT-PCR and CD3 ${ }^{+}$CD $8+T$ cell flow analysis of 4T1-NEO and 4T1-TGFBR3 tumors. (B) Foxp3 qRT-PCR and CD4+FOXP3+ Treg flow analysis of 4T1-NEO and 4T1-TGFBR3 tumors. (A and B) qRT-PCR, 5 tumors/group, data representative of 3 independent experiments; flow analysis, 9 tumors/ group, data pooled from 3 independent experiments. (C) Cd8 qRT-PCR and CD $3^{+}$CD $8{ }^{+} \mathrm{T}$ cell flow analysis of 4T1-NEO and 4T1-TGFBR3 TDLNs. 8 mice/group. Pooled from 2 independent experiments. (D) Foxp3 qRT-PCR and FOXP3 ${ }^{+}$IHC of 4T1-NEO and 4T1-TGFBR3 TDLNs. qRT-PCR, 8 mice/group, data pooled from 2 independent experiments; IHC, 5 random $\times 40$ fields/condition, data representative of 2 independent experiments. (E) Allogeneic T cell proliferation assays using distant LN and TDLN tissues resected from 4T1-NEO and 4T1-TGFBR3 tumor-bearing mice. 5 mice/group. Representative of 2 independent experiments. (F) HER2/ NEU-specific autologous T cell proliferation assays using 4T1-NEO-HER2 and 4T1-TGFBR3-HER2 TDLNs. 5 mice/group. Representative of 2 independent experiments. (G) HER2/NEU-specific IFN- $\gamma$ ELISPOT assay of 4T1-NEO-HER2 and 4T1-TGFBR3-HER2 TDLNs. 5-10 mice/group. Performed in triplicate. Flow cytometry data are expressed as a percentage of viable total tumor or TDLN cell number. Data are mean \pm SEM. ${ }^{\star} P<0.05,{ }^{*} P<0.005,{ }^{* *} P<0.0005,2$-tailed Student's $t$ test.

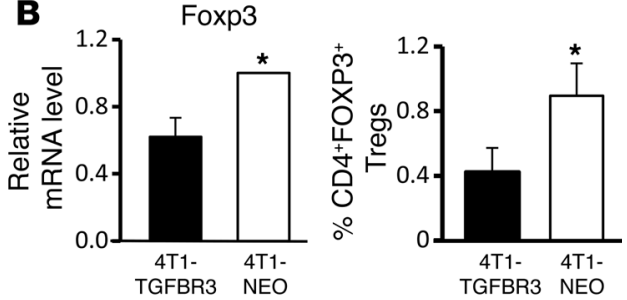

4T1-TGFBR3-HER2, respectively; Supplemental Figure 1, $B$ and $C$ ) and implanted them into syngeneic female WT and NSG mice. While 4T1-NEOHER2 and 4T1-TGFBR3HER2 tumor incidence was uniformly high in NSG hosts, 4T1-TGFBR3-HER2 tumor incidence in WT mice was significantly reduced compared with 4T1-NEO-HER 2 control tumors (Figure 1C). Consistent with the results obtained with the parental cell lines, whereas the 4T1-NEOHER2/4T1-TGFBR3-HER2 primary tumor volume ratio was elevated in WT mice, this ratio was approximately 1 in the NSG strain (Figure 1C). We subsequently investigated potential crosstalk between the TGFBR3 and HER2/ NEU signaling pathways and failed to observe any changes in cell proliferation in vivo or apoptosis in vitro (Supplemental Figure 3). However, 4T1-TGFBR3-HER2 tumors demonstrated increased $\mathrm{CD}^{+}$ $\mathrm{T}$ cell infiltration compared with 4T1-NEO-HER2 tumors (Figure 1D), which suggests that the differences in tumor growth may be secondary to the enhanced generation of antitumor immunity in 4T1-TGFBR3 tumors. Consistent with our hypothesis that TGFBR3 regulates the antitumor immune response, there were no significant differences in tumor cell apoptosis in NSG hosts, whereas mice (NOD/SCID gamma; referred to herein as NSG mice). Similar to previous findings, TGFBR3 expression was consistently found to suppress $4 \mathrm{~T} 1$ metastasis in WT mice (Supplemental Figure 2, A-C). However, 4T1-TGFBR3 and 4T1-NEO tumors grew more rapidly in the NSG strain than in WT hosts (Figure 1A), demonstrating an important role for the immune system in regulating the primary tumor growth of this breast cancer model. While the ratio of overall tumor burden in 4T1-NEO tumor-bearing mice to that in 4T1-TGFBR3 tumor-bearing mice approached unity in NSG hosts after primary tumor resection, the 4T1-NEO/4T1-TGFBR3 ratio exceeded 3 in WT hosts (Figure 1B). These data supported a role for an intact immune system in TGFBR3-mediated suppression of breast cancer progression. To monitor tumor-associated antigen-specific $\mathrm{T}$ cells, we generated HER2/NEU-expressing 4T1-NEO and 4T1-TGFBR3 tumor cell lines (4T1-NEO-HER2 and tumor cell apoptosis in vivo was increased in 4T1-TGFBR3-HER2 versus 4T1-NEO-HER2 tumors in WT mice (Figure 1, E and F). These findings suggested that TGFBR3 elicits a proapoptotic $\mathrm{T}$ cell-mediated immune response. Indeed, 4T1-TGFBR3 tumor tissues exhibited both increased $\mathrm{CD} 3^{+} \mathrm{CD} 8^{+}$cytotoxic $\mathrm{T}$ cell infiltration and diminished $\mathrm{CD} 4^{+} \mathrm{FOXP}^{+}$Treg infiltration compared with 4T1-NEO tumors (Figure 2, A and B). 4T1-NEO tumors also demonstrated Th2 polarization relative to 4T1-TGFBR3 tumors, with elevated levels of $I l 4, I l 13$, and Il10 mRNA along with a modest decrease in Ifng (Supplemental Figure 4), further indicating that the loss of TGFBR3 supports the development of an immune microenvironment conducive to tumor growth.

TDLNs serve a critical role in the generation of host antitumor immunity. Similar to the results in primary tumor tissues, TDLNs of 4T1-TGFBR3 tumors contained increased numbers of $\mathrm{CD}^{+}$ 
A

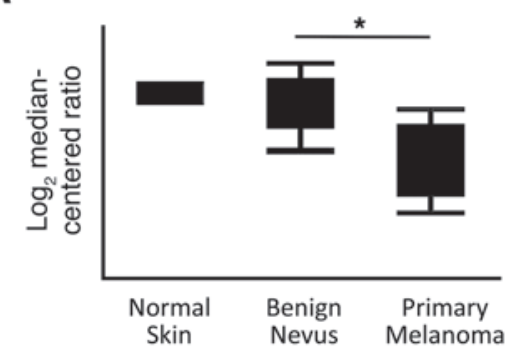

D

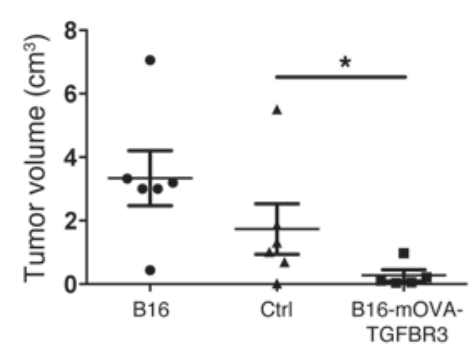

B

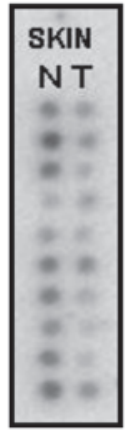

C
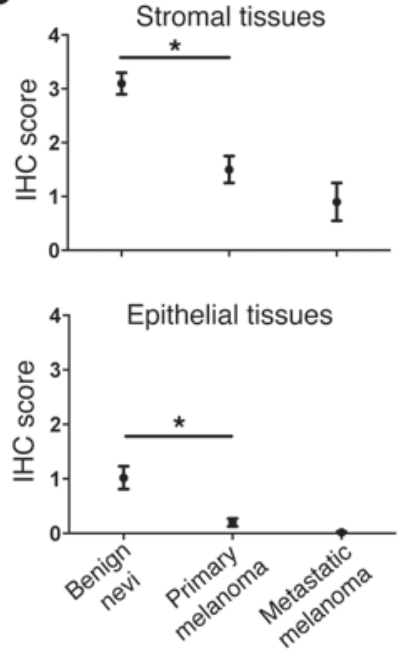

Benign Nevus

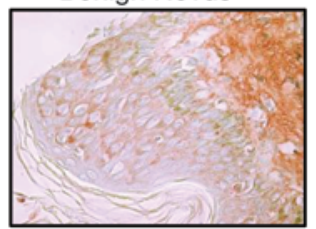

Metastatic melanoma

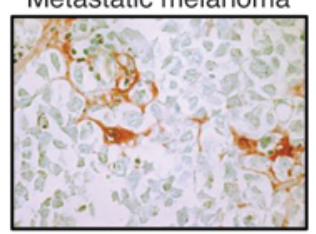

$\mathbf{F}$

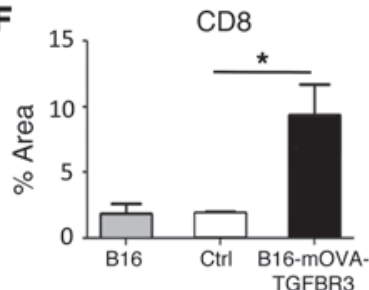

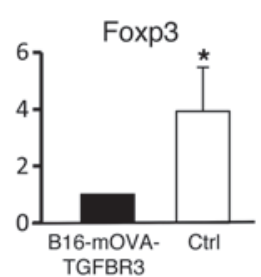

TGFBR3
Cd8

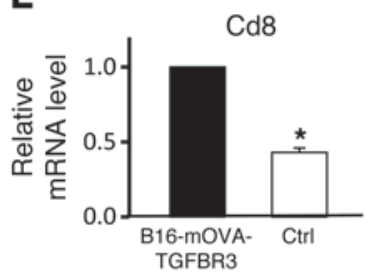

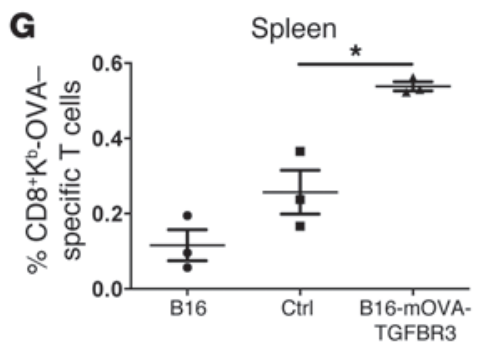

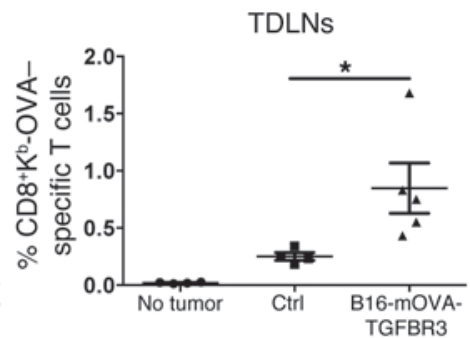

Figure 3

Loss of TGFBR3 occurs during melanoma progression and suppresses the development of antitumor immunity. (A) Oncomine microarray TGFBR3 expression analysis in human benign nevi and primary melanoma tissues. See Supplemental Table 2. (B) DNA hybridization blot analysis of TGFBR3 mRNA levels in normal human skin (N) and melanoma tumor tissues (T). (C) TGFBR3 IHC of human benign nevi, primary melanoma, and metastatic melanoma tumor tissues. 100 core tissues were evaluated. Representative $\times 20$ fields are also shown. (D) B16-mOVATGFBR3 tumor growth relative to B16-mOVA control (ctrl) and B16/F10 (B16) tumors in syngeneic hosts. 5-6 tumors/condition. Representative of 3 independent experiments. (E) qRT-PCR of Cd8 and Foxp3 in B16-mOVA-TGFBR3 versus B16-mOVA tumors. 3 tumors/condition. Representative of 2 independent experiments. (F) CD8 and FOXP3 IHC of B16-mOVA-TGFBR3, B16-mOVA, and B16/F10 tumors. 10 fields/tumor, 3 tumors/ condition. (G) $\mathrm{K}^{\mathrm{b}-O V A_{257-264}-\text { specific CD8 }}{ }^{+} \mathrm{T}$ cell tetramer analysis of resected splenic and TDLN tissues from B16-mOVA-TGFBR3, B16-mOVA, and B16/F10 tumor-bearing mice. 3-6 mice/group. Representative of 2 independent experiments. Data are mean \pm SEM. ${ }^{*} P<0.05,2$-tailed Student's $t$ test (A, E, and F), 1-way ANOVA (C, D, and $\mathbf{G})$.

cytotoxic $\mathrm{T}$ cells and diminished Treg populations compared with 4T1-NEO TDLNs (Figure 2, C and D, and Supplemental Figure 5). These properties were consistent with the enhanced $\mathrm{T}$ cell proliferative capacity of APCs observed in 4T1-TGFBR3 TDLNs (Figure $2 \mathrm{E})$. Further illustrating the locoregional effects of tumor-mediated immunosuppression in this tumor model, we also observed an increase in $\mathrm{T}$ cell proliferative responses in distant $\mathrm{LN}$ tissues relative to TDLN tissues (Supplemental Figure 6A). Finally, 4T1-TGFBR3 TDLNs also consistently generated more robust HER2/NEU anti- gen-specific T cell responses than did 4T1-NEO TDLNs (Figure 2, F and G, and Supplemental Figure 6B). To further test our hypothesis, we silenced TGFBR3 expression in a different murine mammary carcinoma cell line in the absence of an exogenous antigen (66CL4 cells) and examined the effects on the local immune microenvironment after implanting these cells in the mammary fat pad of $\mathrm{BALB} / \mathrm{c}$ mice (Supplemental Figure 7, A and B). Consistent with our previous data, we observed a significant decrease in the CD8/FOXP3 tumor-infiltrating lymphocyte ratio upon TGFBR3 downregulation 
A

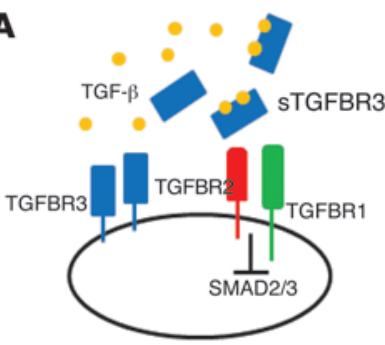

Tumor cell

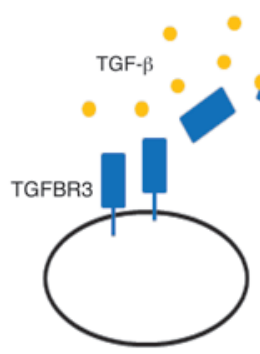

Tumor cell

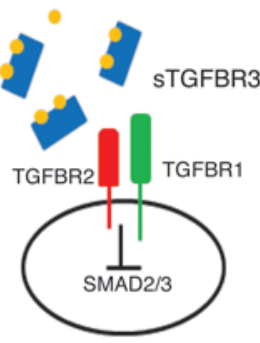

Stromal cell

B
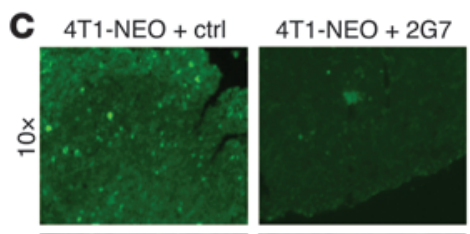

4T1-TGFBR3
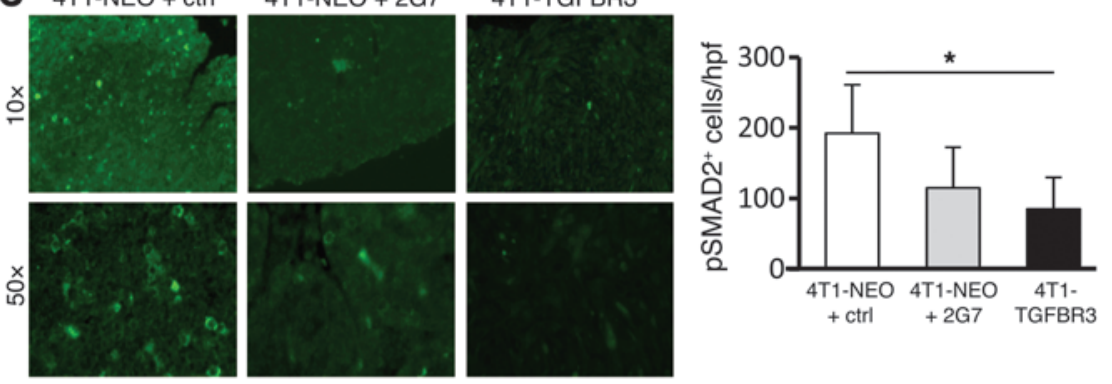

pSMAD2

E

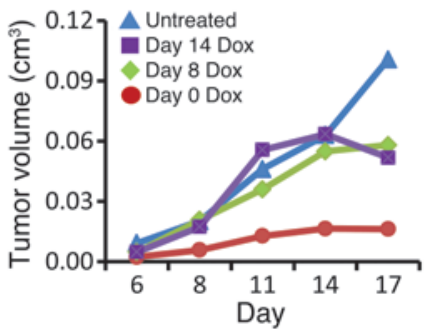

$\mathbf{F}$

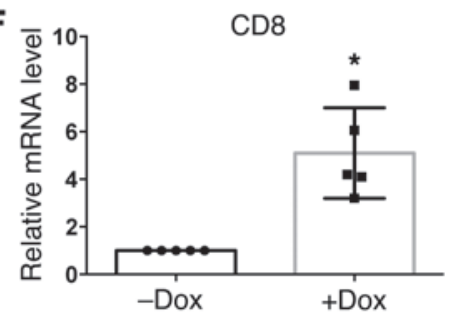

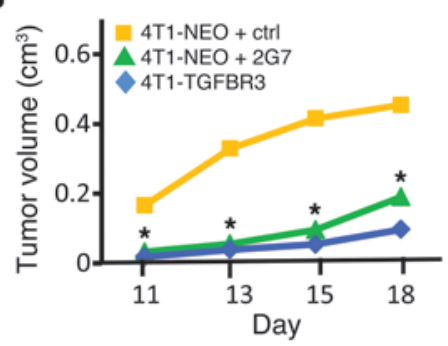

D

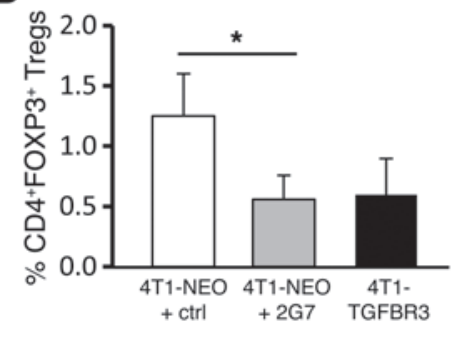

G

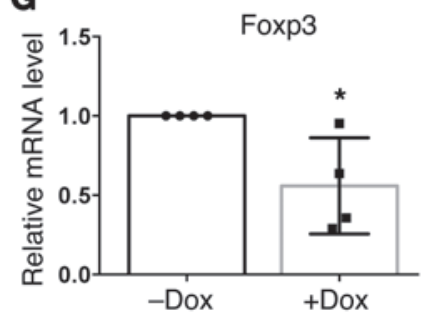

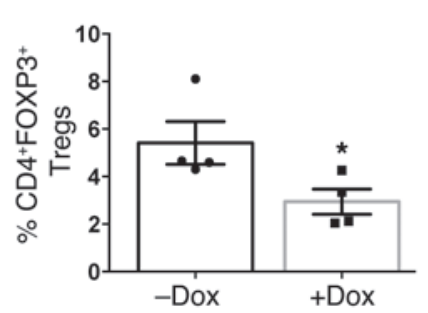

H

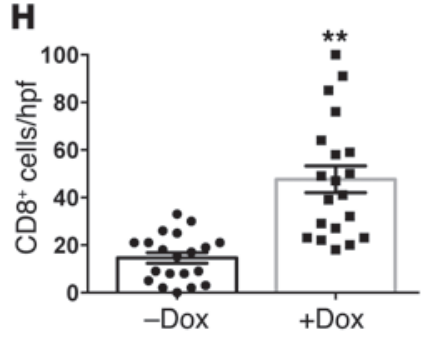

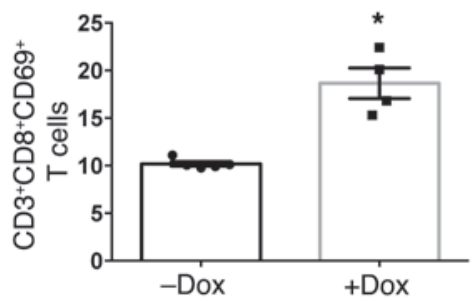

Figure 4

Sequestration of TGF- $\beta$ by sTGFBR3 modulates the tumor microenvironment. (A) Left: sTGFBR3 inhibited downstream TGF- $\beta$-mediated signaling in tumor cells in an autocrine manner. Right: sTGFBR3 also inhibited TGF- $\beta$ signaling in local stromal cells in a paracrine manner. (B) Tumor growth measurements with and without TGF- $\beta$-targeted blockade. $2 \mathrm{G} 7$, anti-pan-TGF- $\beta$ mAb. 8 mice/group. (C) Immunofluorescence analysis of pSMAD2 in TDLN tissues. 5 random $\times 10$ fields across $3-4 \mathrm{LNs}$ /condition (representative $\times 10$ and $\times 50$ images are also shown). Representative of 2 independent experiments. (D) CD4+FOXP3+ Treg flow cytometry of TDLN tissues. 4 mice/group. (E) Doxycycline (Dox) treatment was initiated at different times after 4T1-sTGFBR3 ${ }_{\text {Tet }}$ tumor implantation, and mice were monitored for tumor progression. $(\mathbf{F})$ Cd8 qRT-PCR analysis of primary 4 T1-sTGFBR3 ${ }_{\text {Tet }}$ tumors with or without doxycycline. Flow cytometry analysis of activated CD8 ${ }^{+}$T cells in 4 T1-sTGFBR3 ${ }_{\text {Tet }}$ TDLNs is also shown. (G) Foxp3 qRT-PCR analysis of primary 4T1-sTGFBR3 ${ }_{T e t}$ tumors with or without doxycycline. Flow cytometry analysis of CD4+FOXP3 ${ }^{+}$

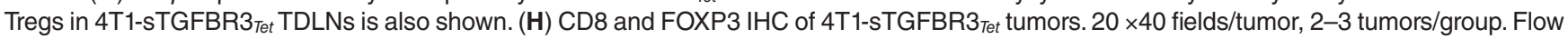
cytometry data are expressed as a percentage of viable total tumor cell number. Data are mean \pm SEM. ${ }^{*} P<0.05$, ${ }^{* *} P<0.005$, 2-tailed Student's $t$ test (B and $\mathbf{E}-\mathbf{G})$, 1-way ANOVA (C and $\mathbf{D}$ ), Mann-Whitney $U$ test $(\mathbf{H})$.

(Supplemental Figure 7, C and D). Overall, these data suggest that loss of tumor-expressed TGFBR3 during cancer progression dampens the $\mathrm{T}$ cell antitumor immune response, thereby promoting the development of an immunotolerant microenvironment.
Loss of TGFBR 3 occurs during melanoma progression and suppresses the development of antitumor immunity. To investigate whether TGFBR3 had a more general role in regulating the immune response during cancer progression, we explored TGFBR3 function in melanoma 

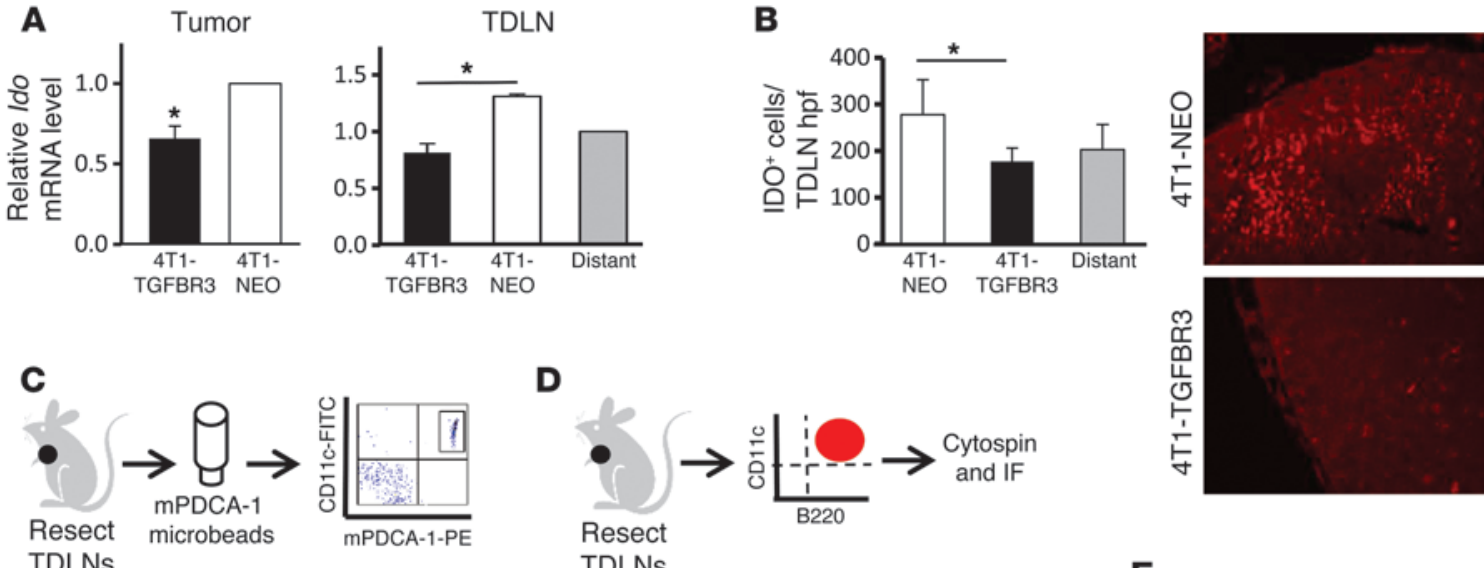

D
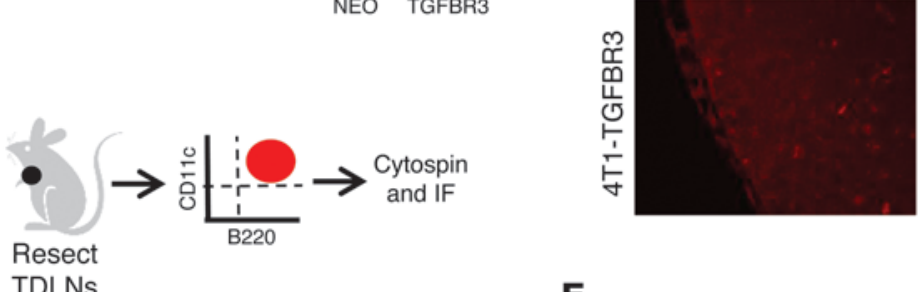

TDLNs

TDLNs
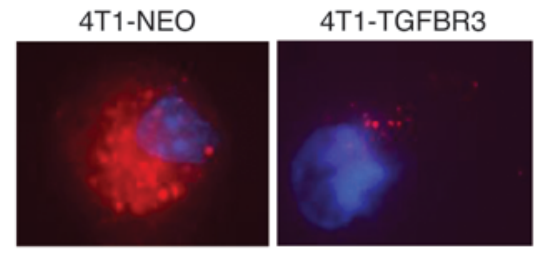

E

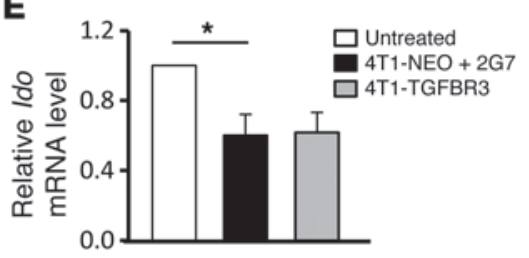

F

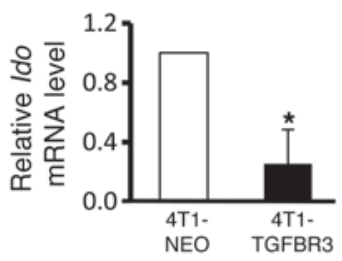

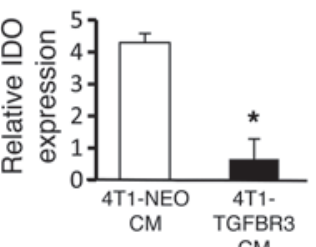

$\mathrm{CM}$

H

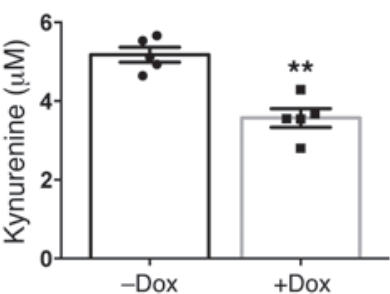

I

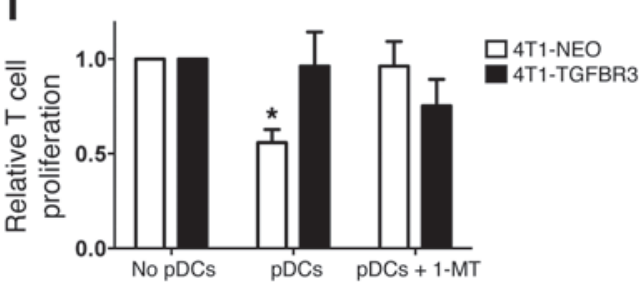

Figure 5

Tumor-derived sTGFBR3 inhibits expression and enzymatic activity of IDO by local pDC populations within the tumor microenvironment. (A) Ido qRT-PCR analysis of 4T1-NEO and 4T1-TGFBR3 tumors and TDLNs. (B) IDO immunofluorescence of TDLNs. Representative $\times 20$ images are also shown. (C) TDLN pDC Ido qRT-PCR. Pooled from 3 independent experiments. (D) TDLN pDC IDO immunofluorescence. Representative $\times 100$ images of pDCs isolated in 2 independent experiments are also shown. Isotype controls showed no staining. (E) Ido qRT-PCR analysis of 4T1-NEO and 4T1-TGFBR3 TDLNs after 2G7 treatment. 6 mice/group. (F) In-cell Western blot (left; pooled from 3 independent experiments) and traditional Western blot (right; representative of 2 independent experiments) of pDC IDO expression in the presence or absence of 4T1-NEO or 4T1-TGFBR3 CM. IFN- $\gamma$ served as a positive control. (G) pDC-derived IDO enzymatic activity, measured after coincubation with 4T1-NEO or 4T1-TGFBR3 CM. sTGFBR3 served as a positive control. Pooled from 3 independent experiments. (H) Whole 4T1-sTGFBR3 ${ }_{\text {Tet }}$ TDLN IDO enzymatic assay with or without doxycycline. (I) 4T1-NEO and 4T1-TGFBR3 TDLN-derived pDC mixed lymphocyte proliferation assay. Representative of 2 independent assays. Data are mean \pm SEM. ${ }^{\star} P<0.05,{ }^{\star *} P<0.005,2$-tailed Student's $t$ test.

pathogenesis. TGF- $\beta$ signaling is known to promote melanoma metastasis, and elevated TGF- $\beta$ expression during melanoma progression correlates with a poor clinical prognosis $(29,30)$. Similar to our findings in breast cancer, we observed downregulation of TGFBR3 during human melanoma progression at both the mRNA and protein levels (Figure 3, A-C, Supplemental Table 2, and Supplemental Figure 8A), with loss of TGFBR3 expression by both tumor and associated stromal tissues. To understand the potential role of TGFBR3 in regulating the antitumor immune response in melanoma, we implanted TGFBR3-expressing B16membrane ovalbumin (mOVA) melanoma tumor cells (referred to herein as B16-mOVA-TGFBR3 cells) and TGFBR3-nonexpressing B16-mOVA control cells into syngeneic C57BL/6 mice and monitored tumor growth (Supplemental Figure 8B). TGFBR3 expres- 

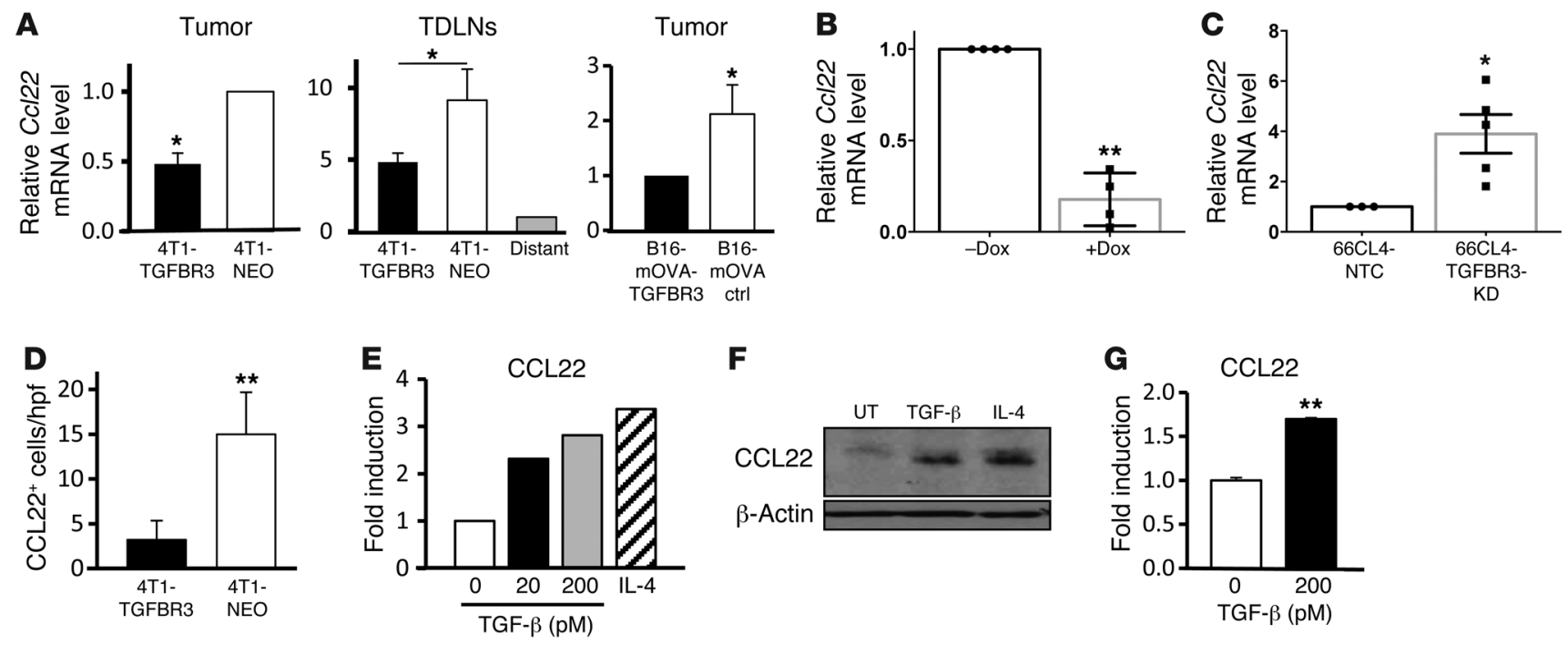

\section{Figure 6}

Loss of TGFBR3 expression in tumors results in enhanced expression of CCL22 by local mDC populations within the tumor microenvironment. (A) Cc/22 qRT-PCR in the indicated tumor and TDLN tissues. 3-5 tumors/group. Representative of 3 independent experiments. (B) Cc/22 qRTPCR in 4T1-sTGFBR3 ${ }_{\text {Tet }}$ tumors in the presence and absence of doxycycline. Representative of 2 independent experiments. (C) Cc/22 qRT-PCR in 66CL4-TGFBR3-KD and 66CL4-NTC tumors. Representative of 3 independent experiments. (D) CCL22 IHC of 4T1-NEO and 4T1-TGFBR3 TDLNs. $10 \times 40$ fields over 3 TDLNs/condition. Representative of 2 independent experiments. (E) Cc/22 qRT-PCR of TGF- $\beta$-treated bone marrow-derived DCs. IL-4 served as a positive control. Representative of 2 independent experiments. (F) CCL22 immunoprecipitation and Western blot of TGF- $\beta$-treated bone marrow-derived DCs. UT, untreated. Representative of 3 independent experiments. (G) CCL22 ELISA of CM after TGF- $\beta$ treatment of bone marrow-derived DCs. Performed in duplicate. Representative of 2 independent experiments. Data are mean \pm SEM. ${ }^{\star} P<0.05,{ }^{\star \star} P<0.005,2$-tailed Student's $t$ test.

sion significantly diminished tumor incidence (0\% vs. $80 \%$ ) and tumor growth in B16-mOVA-TGFBR3 versus B16/F10 and B16mOVA tumors (Figure 3D). Similar to our findings in the $4 \mathrm{~T} 1$ and 66CL4 breast cancer models, B16-mOVA-TGFBR3 tumors exhibited enhanced $\mathrm{CD}^{+} \mathrm{T}$ cell and suppressed $\mathrm{FOXP}^{+}$Treg infiltration compared with B16/F10 and B16-mOVA tumors (Figure 3, E and $\mathrm{F}$, and Supplemental Figure 8D). Importantly, $\mathrm{K}^{\mathrm{b}}$-SIINFEKL tetramer analysis of the spleen and TDLNs revealed increased numbers of $\mathrm{OVA}_{257-264}$-specific $\mathrm{T}$ cell populations in mice bearing B16-mOVA-TGFBR3 versus B16-mOVA tumors (Figure 3G). Together, these data support TGFBR3 as a tumor-expressed modulator of the tumor immune microenvironment in both breast cancer and melanoma.

Loss of TGFBR3 primarily modulates TGF- $\beta$ signaling and results in enhanced expression of IDO and CCL22 by local DC populations within the tumor microenvironment. TGFBR3 is shed from the tumor cell surface to generate sTGFBR3, which in turn sequesters TGF- $\beta$ and suppresses downstream TGF- $\beta$-mediated signaling (Figure $4 \mathrm{~A}$, left) $(27,28)$. As sTGFBR3 can bind several members of the TGF- $\beta$ superfamily (21), we sought to determine the contribution of the TGF- $\beta$ ligands in TGFBR3-mediated immune regulation. We administered the anti-pan-TGF- $\beta$ mAb 2 G7 into the mammary fat pad of female BALB/c mice every 2-3 days after implantation of the 4T1-NEO tumor line (31). Compared with control 4T1-NEO tumors, inhibition of TGF- $\beta$ with the $2 \mathrm{G} 7 \mathrm{mAb}$ recapitulated the diminished 4T1-TGFBR3 tumor size (Figure 4B). These results suggested that loss of TGFBR 3 expression may promote cancer progression by enhancing TGF- $\beta$ signaling within the tumor microenvironment. Consistent with this hypothesis, 2 G7 delivery inhibited TGF- $\beta$ signaling in 4T1-NEO TDLNs to an extent similar to that in 4T1-TGFBR3 TDLNs (Figure 4C). Notably, both the $2 \mathrm{G} 7 \mathrm{mAb}$ and TGFBR3 expression were also associated with decreased levels of $\mathrm{CD}^{+}{ }^{+} \mathrm{FOXP} 3^{+}$Tregs within the TDLNs (Figure 4D). To confirm that sTGFBR3 was indeed responsible for modulating the immunologic responses observed in the 4T1 and B16 tumor models, and that this effect was independent of an exogenous antigen, we generated a tetracycline-responsive 4T1 tumor cell line expressing the ectodomain of TGFBR3 (referred to herein as 4 T1-sTGFBR $3_{\text {Tet }}$ cells; Supplemental Figure 9, A-C). 4T1-sTGFBR $3_{\text {Tet }}$ cells were also transplanted into the mammary fat pads of syngeneic mice before doxycycline was administered at different time points via their water supply. In line with our previous data, doxycycline induction of tumor-mediated sTGFBR3 expression effectively suppressed the growth of this breast cancer model (Figure 4E and Supplemental Figure 2D). Furthermore, the upregulation of sTGFBR3 expression also corresponded with enhanced $\mathrm{CD}^{+} \mathrm{T}$ cell and diminished $\mathrm{FOXP}^{+}$Treg numbers within the $4 \mathrm{~T} 1-\mathrm{sTGFBR} 3_{\text {Tet }}$ tumor microenvironment (Figure 4, F-H, and Supplemental Figure 9, D and E).

DCs orchestrate the activation and modulate the function of different populations of effector T cells. Given that tumor-derived TGFBR 3 regulates both $\mathrm{CD}^{+} \mathrm{T}$ cell and $\mathrm{FOXP}^{+}$Treg populations, we postulated that TGFBR3 tumor expression may modulate local DC function. Consistent with this hypothesis, CD $11 \mathrm{c}^{+} \mathrm{DCs}$ within 4T1-TGFBR3 TDLNs demonstrated a phenotype consistent with enhanced maturation relative to CD11 $\mathrm{c}^{+}$DCs within 4T1-NEO TDLNs (Supplemental Figure 10). Since the phenotype of DCs directly affects DC-dependent $\mathrm{T}$ cell stimulation, these data suggest that the enhanced $T$ cell activation we observed in 4T1-TGFBR3 TDLNs (Figure 2, F and G) may be partially due to diminished 

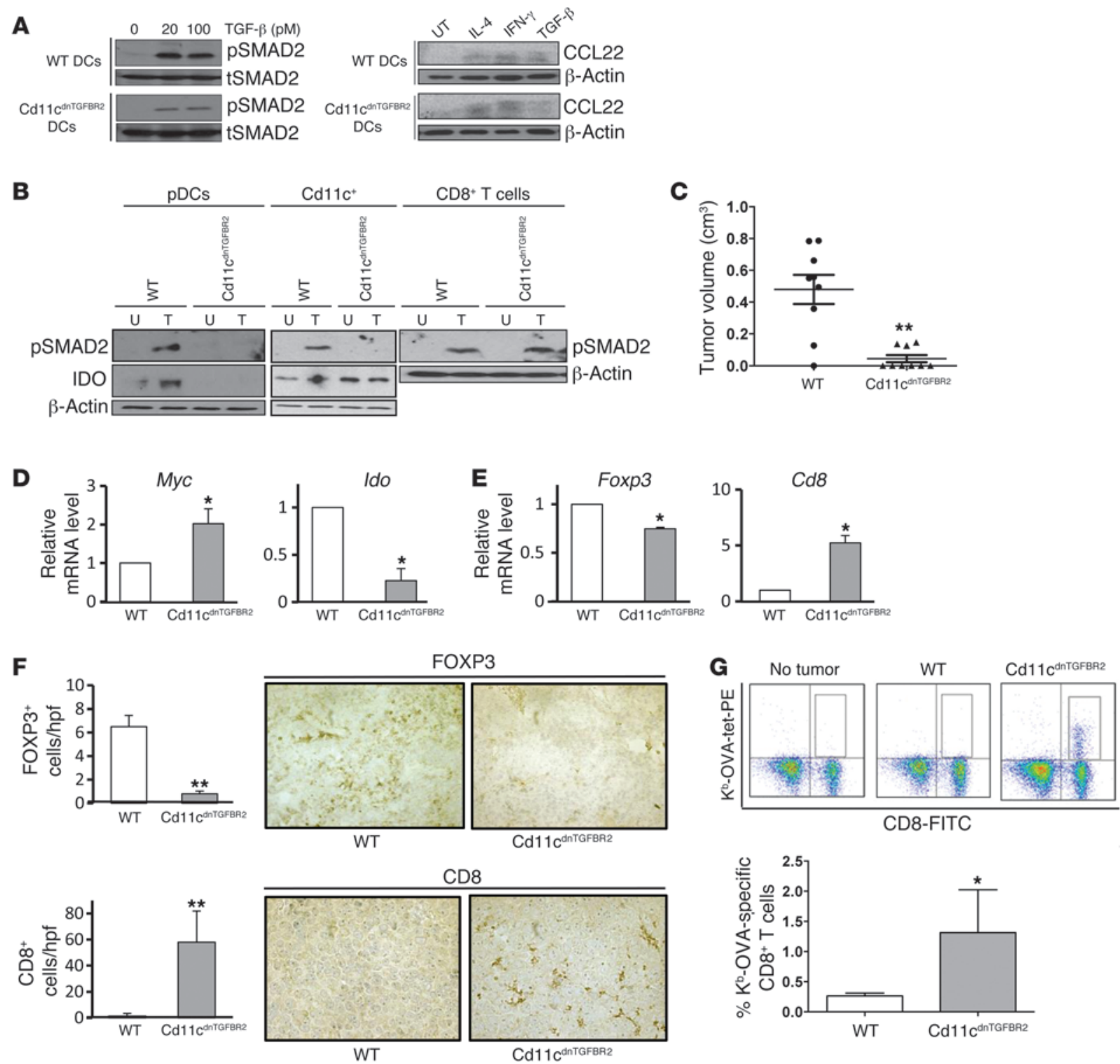

\section{Figure 7}

TGF- $\beta$-mediated suppression of DC function is critical for melanoma tumorigenesis. (A) SMAD2 and CCL22 Western blot analysis of TGF- $\beta$ treated bone marrow-derived DCs isolated from WT and Cd11 $c^{\text {dnTGFBR2 }}$ mice. IL-4 served as a positive control. t-, total. (B) pSMAD2 and IDO Western blot of TGF- $\beta$-treated (T; 20 pM) and untreated (U) splenic PDCA-1+ $p D C s$, splenic CD11 ${ }^{+}$PDCA-1- DCs, and CD8 ${ }^{+}$T cells isolated from WT and Cd11 $c^{\text {dnTGFBR2 }}$ mice. Representative of 2 independent experiments. (C) B16-mOVA tumor volume in WT and Cd11c ${ }^{\text {dnTGFBR2 }}$ mice. 8-9 mice/group. Representative of 2 independent experiments. (D) Myc and Ido qRT-PCR of TDLN pDCs isolated from WT and Cd11 dnTGFBR2 mice. 3 mice/group. Pooled from 2 independent experiments. (E) Foxp3 and Cd8 qRT-PCR of B16-mOVA tumors resected from WT and Cd11cdnTGFBR2 mice. 4 tumors/group. Representative of 2 independent experiments. (F) CD8 and FOXP3 IHC of B16-mOVA tumors resected from WT and Cd11 ${ }^{\text {dnTGFBR2 }}$ mice. 20 random $\times 40$ fields/group (representative $\times 40$ images are also shown). Representative of 2 independent experiments. (G) $\mathrm{K}^{\mathrm{b}}-\mathrm{OVA}_{257-264-\text { specific CD8 }}{ }^{\mathrm{T}} \mathrm{T}$ cell tetramer analysis of splenocytes in B16-mOVA tumor-bearing WT and Cd11c dnTGFBR2 mice. 5 mice/group. Data are mean \pm SEM. ${ }^{\star} P<0.05,{ }^{\star \star} P<0.005$, 1-way ANOVA (C), 2-tailed Student's $t$ test (D-G).

TGF- $\beta$ signaling within these local DC populations. To determine whether tumor-expressed sTGFBR3 can interfere with paracrine DC-specific TGF- $\beta$-mediated signaling, we cocultured 4T1-NEO and 4T1-TGFBR3 tumor conditioned media (CM) with both a murine splenic DC line and purified bone marrow-derived myeloid DCs (mDCs). Similar to the anti-pan-TGF- $\beta$ mAb $2 \mathrm{G} 7$ and recombinant sTGFBR3, 4T1-TGFBR3 CM significantly decreased TGF- $\beta$-induced SMAD2 phosphorylation (pSMAD2) in these DC populations compared with DCs cocultured with 4T1-NEO CM (Supplemental Figure 11, A and B). 4T1-TGFBR3 CM also reversed TGF- $\beta$-induced suppression of phenotypic DC maturation based on CD40 surface expression (Supplemental Figure 11C). Furthermore, in DC coincubation studies, CM from sTGFBR3-expressing COS7 cells recapitulated these findings (Supplemental Figure 11, $\mathrm{D}$ and $\mathrm{E}$ ), thereby establishing tumor cell-expressed sTGFBR3 as an inhibitor of the TGF- $\beta$ signaling pathway in DCs. Collectively, 
A
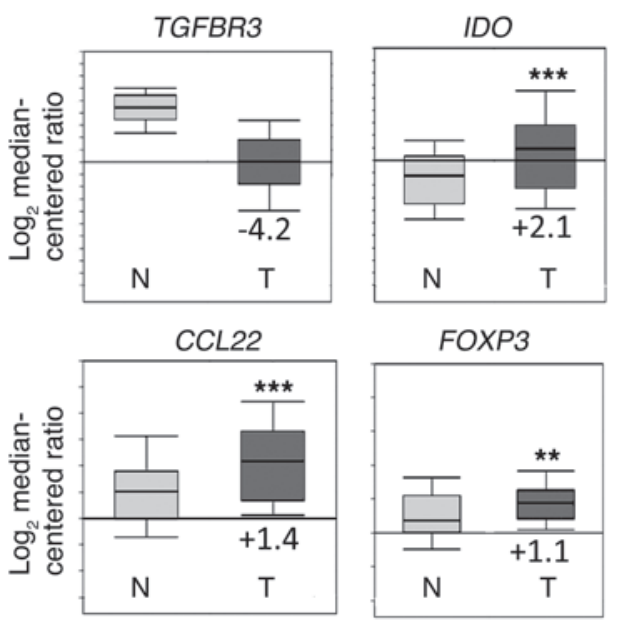

B
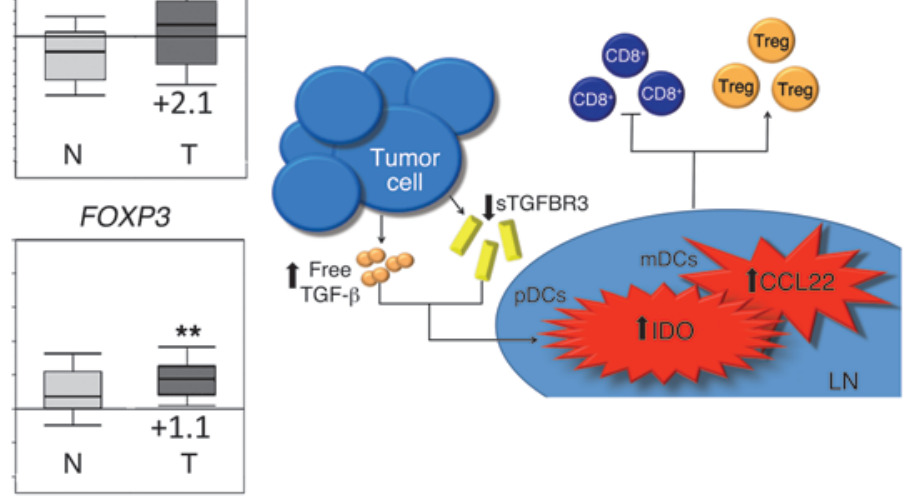

C
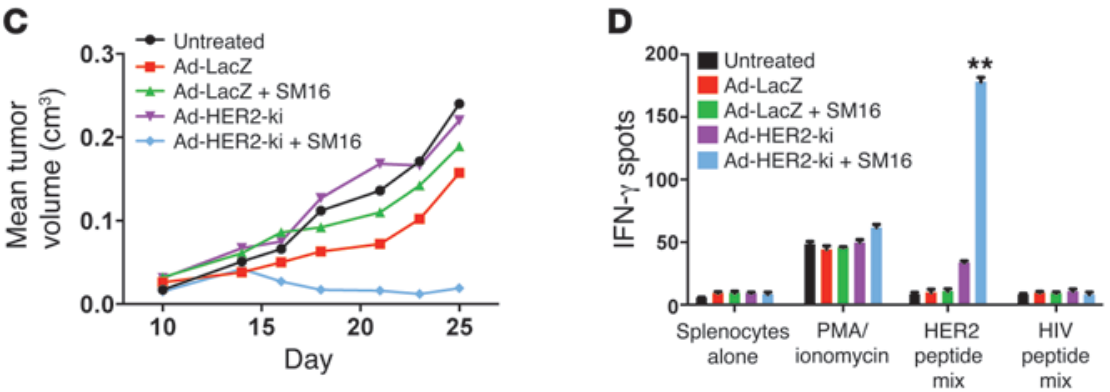

Figure 8

Translational implications of TGFBR3-dependent regulation of antitumor immunity in breast cancer. (A) Oncomine microarray expression analysis of TGFBR3, IDO, CCL22, and FOXP3 in human normal mammary tissues $(\mathrm{N})$ relative to human breast cancers $(T)$. Fold changes in gene expression relative to normal mammary tissues are indicated. See Supplemental Table 2. (B) Loss of TGFBR3 by tumor cells enhances TGF- $\beta$-mediated upregulation of IDO and CCL22 by local pDC and $\mathrm{mDC}$ populations, respectively, resulting in enhanced Treg activation and recruitment. (C) TGF- $\beta$ signaling inhibition in combination with HER2/ NEU vaccination in a HER2/NEU-expressing 4T1 mammary carcinoma model. AdLacZ was used as control vector. 8 mice/group. Representative of 2 independent experiments. (D) IFN- $\gamma$ ELISPOT analysis of HER2/ NEU-specific T cell responses. PMA/ionomycin served as a positive control; HIV peptide mix served as an irrelevant peptide negative control. 3 mice/group. Representative of 2 independent experiments. ${ }^{* \star} P<0.005$, ${ }^{* * *} P<0.0005$, 2-tailed Student's $t$ test.

these results suggest that the production of sTGFBR 3 by tumor tissues is capable of suppressing paracrine TGF- $\beta$-mediated signaling in local APCs (Figure 4A, right).

Consistent with the hypothesis that loss of tumor TGFBR3 expression during cancer progression enhances downstream TGF- $\beta$ signaling in local DCs compared with tumors expressing TGFBR3, the stromal cells of 4T1-NEO tumors, 4T1-NEO TDLNs, and B16mOVA tumors expressed higher mRNA and protein levels of the TGF- $\beta$-responsive immunoregulatory enzyme IDO (Figure 5, $A$ and B, and Supplemental Figure 12). Similar to a previous report (16), IDO was expressed primarily by pDCs within these TDLN tissues (Supplemental Figure 13A). pDCs isolated from 4T1-NEO TDLNs expressed higher mRNA and protein levels of IDO compared with those from 4T1-TGFBR3 TDLNs (Figure 5, C and D). Furthermore, TGF- $\beta$ treatment of murine bone marrow-derived pDCs and splenic pDCs, but not mDCs, robustly upregulated mRNA and protein levels of IDO (Supplemental Figure 13, B and C). In support of the observed relationship between TGFBR3 and IDO expression in the 4T1 and B16 tumor models, delivery of $2 \mathrm{G} 7$ into the $4 \mathrm{~T} 1$ tumor bed of syngeneic mice also decreased IDO expression in nearby TDLN tissues (Figure $5 \mathrm{E})$. In addition, 4T1-TGFBR3 CM suppressed IDO expression by purified splenic pDCs (Figure 5F). Since the enzymatic activity of IDO is critical for its immunoregulatory role, we also investigated the effects of 4T1-TGFBR3 CM on the functional activity of this enzyme. Reflecting the changes observed in IDO expression, 4T1-TGFBR3 CM also suppressed the enzymatic activity of IDO (Figure 5G). Importantly, we also observed a modest but significant suppression of IDO enzymatic activity in whole TDLN tissues resected from 4T1-sTGF$\mathrm{BR} 3_{\text {Tet }}$ tumor-bearing mice upon doxycycline-induced sTGFBR3 expression (Figure $5 \mathrm{H})$. Finally, to demonstrate the differential immunosuppressive function of $\mathrm{pDCs}$ in 4T1-TGFBR3 and 4T1-NEO TDLNs, we tested the ability of these TDLN-derived purified pDCs to inhibit $\mathrm{T}$ cell proliferation in a mixed lymphocyte response assay. We found that pDCs isolated from 4T1-NEO TDLNs, but not 4T1-TGFBR3 TDLNs, robustly suppressed $\mathrm{T}$ cell proliferation (Figure 5I). Moreover, the T cell-suppressive effect of $\mathrm{pDCs}$ was reversed with the IDO inhibitor 1-methyl-DL-tryptophan (1-MT), which suggests that IDO activity plays a critical role in pDCdependent inhibition of $\mathrm{T}$ cell activity. These results demonstrate that tumor-derived TGFBR3 regulates the immune microenvironment through its modulation of $\mathrm{PDC}$-derived IDO.

In addition to IDO, tumors use other Treg-dependent mechanisms to suppress the generation of host immunity. The CCL22 chemokine is primarily expressed by myeloid APCs and promotes the recruitment of CCR4-expressing Treg populations $(32,33)$. Consistent with our observation of increased tumor and TDLNresident Tregs upon loss of TGFBR3 in the murine breast cancer and melanoma models, Ccl 22 mRNA levels were increased in both tumor and TDLN tissues in the absence of tumor-derived TGFBR3 (Figure 6A). These alterations in Ccl22 expression were mediated by sTGFBR3, since doxycycline delivery suppressed Ccl22 mRNA expression in 4T1-sTGFBR3 $3_{\text {Tet }}$ tumors (Figure 6B). We also found reciprocal increases in Ccl22 expression upon TGFBR3 silencing in the 66CL4-TGFBR3-KD tumor model (Figure 6C). These findings were further supported at the pro- 

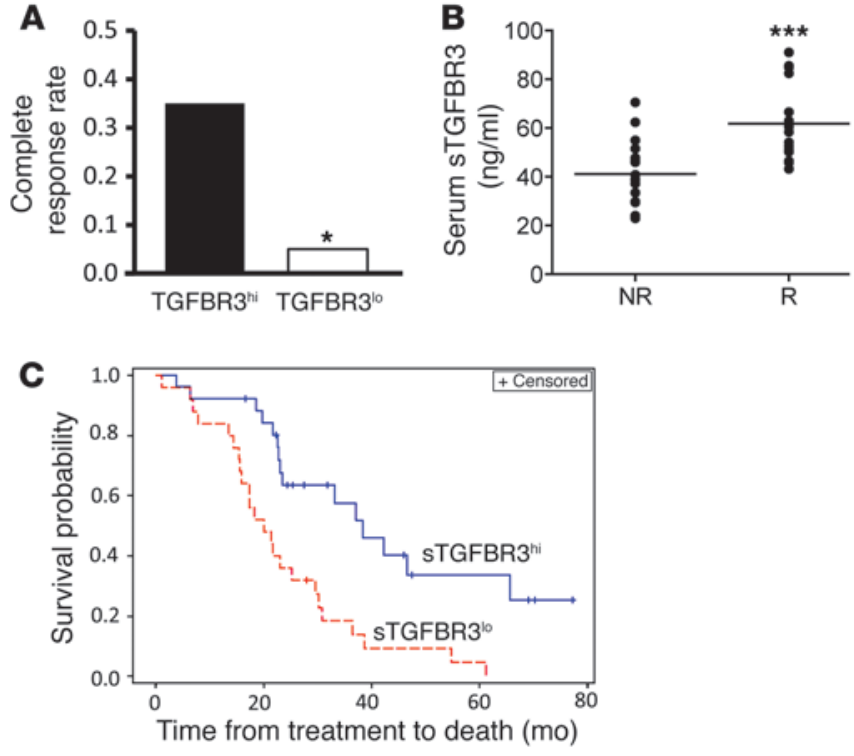

\section{Figure 9}

Translational implications of TGFBR3-dependent regulation of antitumor immunity in melanoma. (A) Stage III melanoma TGFBR3 expression levels were determined by microarray analysis. Complete response rates to ILI therapy by patients with the highest 20\% TGFBR3 expression (TGFBR3 ${ }^{\text {hi }}$ ) and lowest $20 \%$ expression (TGFBR3 ${ }^{\text {lo }}$ ) are shown. 103 patients. (B) sTGFBR3 plasma levels in nonresponding (NR) versus responding (R) stage III melanoma patients. 52 patients. (C) Multivariate survival analysis of stage III melanoma patients according to sTGFBR3 plasma levels. Kaplan-Meier survival curve. Data are mean \pm SEM. ${ }^{*} P<0.05,{ }^{* *} P<0.0005$, Spearman correlation calculation $(\mathbf{A})$, Mann-Whitney $U$ test $(\mathbf{B})$, multivariate analysis $(\mathbf{C})$.

tein level by CCL22 immunohistochemistry (IHC; Figure 6D and Supplemental Figure 14A). Previous work has shown mDCs to be the predominant source of CCL22 expression (32). TGF- $\beta$ mediated dose-dependent upregulation of CCL22 expression in $\mathrm{MDC}$ but not pDC populations, similar to IL-4 (Figure 6, E-G, Supplemental Figure 14, B and C, and ref. 33). To better understand the mechanism of CCL22 upregulation, we conducted a promoter sequence analysis, which revealed evidence of a SMAD3/4 binding element (-256/-247), prompting additional luciferase reporter assays that confirmed the ability of TGF- $\beta$ to activate the Ccl22 promoter (Supplemental Figure 14D and data not shown). These data suggested that TGFBR 3 regulates TGF- $\beta$-induced CCL22 expression in $\mathrm{mDCs}$, resulting in an alternative mechanism for tumor-mediated recruitment of Tregs.

Together, our present findings support a model in which progressive loss of TGFBR3 during cancer progression enhances TGF- $\beta$ mediated upregulation of both IDO and CCL22 by distinct DC populations within the tumor microenvironment. We hypothesize that this mechanism, coupled with the elevated levels of TGF- $\beta$ observed in many human cancers, generates an immunotolerant state, further facilitating tumor progression and metastasis.

TGF- $\beta$-mediated suppression of DC function is critical for melanoma tumorigenesis. To determine the importance of DC-specific TGF- $\beta$ signaling in the process of tumor-mediated immunosuppression, we investigated B16 melanoma development in the previously developed $C d 11 c^{d n T G F B R 2}$ transgenic mouse strain, which expresses dominant-negative TGFBR2 (dnTGFBR2) under control of the
Cd11c promoter to ablate the TGF- $\beta$ signaling pathway specifically in DC populations (34-36). As expected, purified bone marrow-derived and splenic pDCs from CD11 $c^{d n T G F B R 2}$ mice exhibited diminished TGF- $\beta$ signaling compared with DCs of WT mice (Figure 7, A and B, and Supplemental Figure 15A). Consistent with dampened TGF- $\beta$ signaling, $C d 11 c^{d n T G F B R 2}$ splenic and bone marrow-derived $\mathrm{mDCs}$ also exhibited decreased TGF- $\beta$-dependent expression of IDO and CCL22, respectively (Figure 7, A and B). In contrast, the TGF- $\beta$ signaling pathway remained mostly intact in splenic $\mathrm{CD}^{+} \mathrm{T}$ and NK cells from Cd11 $c^{d n T G F B R 2}$ mice (Figure 7B, Supplemental Figure 15B, and Supplemental Figure 16), which suggests that TGF- $\beta$-mediated suppression of antitumor immunity is predominantly restricted to direct $\mathrm{T}$ cell and NK cell inhibition in this transgenic host. We next monitored B16-mOVA tumor growth, and found it to be decreased in $C d 11 c^{d n T G F B R 2}$ versus WT hosts (Figure 7C). Although the number of DCs within the tumor microenvironment of $C D 11 c^{d n T G F B R 2}$ mice was not significantly different from the number of TDLN-derived DCs of WT mice (Supplemental Figure 17), TDLN-derived pDCs from $C d 11 c^{d n T G F B R 2}$ mice were compromised in their ability to respond to TGF- $\beta$, based on decreased expression of Ido and increased expression of Myc (Figure 7D). The primary B16-mOVA tumors resected from $C d 11 c^{d n T G F B R 2}$ hosts also demonstrated reduced infiltration of FOXP3 $3^{+}$Tregs and enhanced numbers of $\mathrm{CD}^{+} \mathrm{T}$ cells (Figure $7, \mathrm{E}$ and $\mathrm{F}$ ). These findings were further corroborated by the enhanced generation of OVA-specific $\mathrm{CD}^{+} \mathrm{T}$ cells in the spleens of CD11 $c^{d n T G F B R 2}$ tumor-bearing hosts (Figure 7G). Together, these data support our previous work in both the 4T1 and B16 tumor models and suggest that the DC-specific TGF- $\beta$ signaling pathway is critical for tumor-mediated immunosuppression.

Translational implications of TGFBR3-dependent regulation of antitumor immunity. In human breast cancers, decreased TGFBR3 expression was also accompanied by increased IDO, CCL22, and FOXP3 expression (Figure 8A and Supplemental Table 3), suggesting a role for the loss of TGFBR3 expression and enhanced TGF- $\beta$ signaling in driving tumor-mediated immunosuppression in human breast cancer. Comparable gene expression associations were also detected in human melanoma tissues (Supplemental Figure 18 and Supplemental Table 4). Overall, our data suggest that downregulation of TGFBR3 during cancer progression is accompanied by increased IDO and CCL22 expression by local DCs, promoting both the development and the recruitment of FOXP3 ${ }^{+}$Tregs in the tumor microenvironment (Figure 8B). To further test this hypothesis, we explored whether inhibiting TGF- $\beta$ signaling potentiates the efficacy of cancer vaccine approaches. Treating murine HER2/NEU-expressing 4T1 tumors with either an adenoviral vector expressing a kinase-inactive form of HER2/NEU (AdHER2ki) or with the TGFBR1 serine/threonine kinase inhibitor SM16 had no effect on tumor growth (Figure 8C and refs. 37-40). However,

\section{Table 1}

Melanoma TGFBR3 expression and clinical response to ILI therapy

\begin{tabular}{lcc}
\hline & TGFBR3 $^{\text {hi }}$ & TGFBR3 $^{\text {lo }}$ \\
Complete response $(n)$ & 7 & 1 \\
Progressive disease $(n)$ & 1 & 6 \\
\hline$P=0.0046, x^{2}$ an & &
\end{tabular}

$P=0.0046, \chi^{2}$ analysis. 


\section{Table 2}

Plasma sTGFBR3 levels and overall survival in stage III melanoma patients after ILI therapy

\begin{tabular}{lcc}
\hline Variable & Odds ratio $(\mathbf{9 5} \% \mathbf{~ C I})$ & $\boldsymbol{P}$ \\
sTGFBR3 & $0.66(0.52-0.83)$ & $<0.001$ \\
Age & $0.99(0.87-1.13)$ & 0.893 \\
Stage & $1.17(0.53-2.61)$ & 0.695 \\
Tumor burden $^{\mathrm{A}}$ & $2.83(1.26-6.38)$ & 0.012 \\
\hline
\end{tabular}

AHigh, $>10$ lesions, or 1 lesion $>3 \mathrm{~cm}$ diameter; low, <10 lesions.

the combination of AdHER2ki with SM16 synergistically inhibited tumor growth (Figure 8C). The therapeutic benefit derived from the combination of an antigen-specific vaccine with a TGF- $\beta$ signaling inhibitor was primarily mediated by an enhanced antitumor $\mathrm{T}$ cell response (Figure 8D). These results support the proposed mechanism involving the TGFBR3/TGF- $\beta$-IDO/CCL22-FOXP3 ${ }^{+}$ Treg axis, while providing additional evidence that inhibiting the TGF- $\beta$ signaling pathway in the tumor microenvironment could synergistically enhance the efficacy of tumor-targeted vaccines (41).

These data suggested that TGFBR3 modulates the immune response to melanoma. Therefore, we used gene microarray analysis of resected human tumor tissues as well as ELISA analysis of the corresponding plasma samples of in-transit stage III melanoma patients to investigate the relationship between TGFBR3/ sTGFBR3 and clinical outcome in this patient population (42). After isolated limb infusion (ILI) therapy, patients in the highest quintile of tumor TGFBR3 expression exhibited a significantly higher complete response rate than those within the lowest quintile (Figure 9A and Table 1). Furthermore, plasma sTGFBR3 levels were significantly higher in stage III melanoma patients responding to ILI therapy than in the nonresponding population (Figure 9B). Finally, multivariate analysis showed that patients with plasma sTGFBR3 levels higher than the median demonstrated significantly improved overall survival after treatment (hazard ratio 0.66 , $P<0.0001$; Figure 9C, Table 2, and Supplemental Figure 19). These data suggest that the loss of TGFBR3 during melanoma progression may suppress antitumor immunity and that plasma levels of sTGFBR3 may serve as a useful prognostic and/or immunotherapeutic predictive biomarker in melanoma patients.

\section{Discussion}

Here, we describe a novel mechanism by which loss of TGFBR3 expression effectively dampens the host antitumor immune response. As we have previously demonstrated, TGFBR3 is frequently downregulated during cancer progression and suppresses tumor cell invasion and metastasis in a cell-autonomous manner $(25,26)$. Our present observations supported our prior data while expanding on these findings, as loss of TGFBR3 expression also resulted in enhanced TGF- $\beta$-dependent signaling within the tumor microenvironment, thereby suppressing the development of tumor antigenspecific immune responses in both breast cancer and melanoma.

Specifically, we have shown that diminished TGFBR3 expression by progressing breast and melanoma tumors was associated with a decrease in $\mathrm{CD}^{+} \mathrm{T}$ cell and an increase in $\mathrm{FOXP}^{+}$Treg populations in the tumor microenvironment, based on quantitative RTPCR (qRT-PCR) analysis, flow cytometry, and IHC. Furthermore, decreased tumor-dependent TGFBR3 expression correlated with suppressed tumor-associated antigen-specific $\mathrm{T}$ cell responses, based on $\mathrm{T}$ cell proliferation assays, IFN- $\gamma$ ELISPOT studies, and tetramer flow analysis.

Given the critical role of DCs in directing $\mathrm{T}$ cell activation and differentiation, we focused our studies on local DCs within the tumor microenvironment and found that loss of TGFBR3 expression significantly affected the function of local DC populations within both the primary tumor and TDLN tissues. A decrease in tumor-associated TGFBR3 expression augmented TGF- $\beta$-dependent upregulation of IDO expression and enzymatic activity in pDCs and CCL22 expression in mDCs. These mechanistic studies further highlighted the substantial differences in TGF- $\beta$ responsiveness and TGF- $\beta$ signaling between $\mathrm{mDC}$ and $\mathrm{PDC}$ populations. Both of these alterations in the tumor immune microenvironment supported enhanced Treg infiltration within the tumor bed and contributed to the development of an immunotolerant site. Tumors suppressed the generation of local immune responses by commandeering the IDO- and CCL22-dependent mechanisms of Treg generation and recruitment, which suggests that loss of TGFBR3 expression is an important, relatively early mechanism for establishing the equilibrium phase of the proposed cancer immunoediting model $(3,22,43)$.

Our data demonstrated that IDO expression levels and enzymatic function were enhanced in tumor tissues exhibiting TGFBR3 downregulation. These results were consistent with previous studies suggesting that TGF- $\beta$ is a major regulator of IDO-mediated long-term immunotolerance in the tumor microenvironment (11). Although other factors have been shown to induce the upregulation of IDO expression by specific DC subsets, such as CD40-CD40L and CTLA-4-CD80/CD86 ligation in the context of $\mathrm{CD}^{+}{ }^{+} \mathrm{T}$ cells, the elevated TGF- $\beta$ levels within the tissue microenvironment of the tumor may dominate local IDO signaling, given that the TGF- $\beta$ cytokine can downregulate many of these pathways $(8,13,44-46)$. This further emphasizes the potential importance of tumor-mediated regulation of TGF- $\beta$ signaling. In addition, we showed that $\mathrm{pDC}$-dependent expression of IDO was critical for suppressing local $\mathrm{T}$ cell responses and that this effect was enhanced upon loss of TGFBR3. Our demonstration that TGF- $\beta$ induced the upregulation of CCL22 expression was consistent with recently published findings in hepatocellular carcinoma (47). However, our present data suggest that TGF- $\beta$ is also capable of directly activating the Ccl22 promoter via a SMADdependent mechanism. Although the roles of these factors have been described in tumor immunoevasion, the regulatory pathway by which cancers manipulate their expression and function in the immune microenvironment have remained unclear. Our present study provides insight into a single unifying mechanism that modifies the function of both IDO and CCL22, while also promoting the intrinsic property of tumor cell migration and invasion.

While this work is largely based on murine cancer models that have been genetically altered to restore TGFBR3 expression, the 4T1-TGFBR3 tumor cell line expresses only modest levels of TGFBR3 relative to the endogenous TGFBR3 expression of other murine breast cancer cell lines (Supplemental Figure 20), which argues against overexpression artifacts (22). Furthermore, silencing endogenous TGFBR3 expression in the related 66CL4 murine mammary carcinoma cell line induced reciprocal changes in $\mathrm{CD}^{+} \mathrm{T}$ cell and $\mathrm{FOXP}^{+}$Treg tumor infiltration (Supplemental Figure 7), consistent with our observations in the 4T1 tumor model. These findings argue that physiological downregulation 
of TGFBR3, which has been shown to occur at relatively early time points in human breast cancer development (22), is capable of modulating the immune microenvironment.

In addition to demonstrating the effect of TGFBR3 on local $T$ cell responses, we also showed that DC-specific TGF- $\beta$ signaling was critical for melanoma progression and implicated this pathway as an important modulator of antitumor $\mathrm{T}$ cell responses (Figure 7). These results were consistent with recent findings showing that DC-specific ablation of TGFBR2 results in multiorgan autoimmunity (48). Using both in vitro and in vivo data, we demonstrated that sTGFBR3 was essential for sequestering TGF- $\beta$ within the tumor bed and suppressing both IDO and CCL22 expression by local $\mathrm{pDC}$ and $\mathrm{mDC}$ populations, respectively (Figures 4-6). Further supporting the validity of these studies, our microarray data derived from human breast cancer (Figure 8A) and melanoma tissues (Supplemental Figure 18) as well as clinical data from melanoma patients (Figure 9) showed additional evidence for a mechanistic connection among TGFBR3, the expression of IDO and CCL22, and tumor infiltration by FOXP3 ${ }^{+}$Tregs.

Importantly, by generating compelling evidence that inhibiting the TGF- $\beta$ signaling cascade within the tumor microenvironment could result in the generation of a more robust $\mathrm{T}$ cell-mediated antitumor immune response (49), the present study provides an intellectual framework for targeting TGF- $\beta$ signaling in the context of cancer immunotherapy (41). Indeed, by coupling the TGFBR1 inhibitor SM16 with a HER2/NEU vaccine in a murine breast cancer model, we demonstrated that the antitumor immune response could be synergistically enhanced (Figure 8, C and D). As toxicity concerns regarding these agents represent a major hurdle in inhibiting TGF- $\beta$ signaling (50), specifically targeting TGF- $\beta$ signaling in pDCs may provide a strategy for reversing TGF- $\beta$ dependent tumor-mediated immunosuppression while minimizing potential systemic side effects.

Our work also showed that sTGFBR3 levels could be measured in plasma and may serve as a prognostic and predictive biomarker for patients with advanced melanoma. Indeed, our data suggest that in-transit melanoma TGFBR3 expression as well as plasma sTGFBR3 levels correlated with clinical response rates to ILI therapy using cytotoxic chemotherapy. Furthermore, patients with plasma sTGFBR3 levels above the median exhibit superior overall survival compared with their counterparts with lower plasma sTGFBR3 levels. Based on the TGF- $\beta$ sequestration model, elevated levels of sTGFBR3 in the plasma may correlate with lower levels of TGF- $\beta$ ligand in the tumor microenvironment and improved responses to immunotherapy. We propose that these experimental observations may be due to sTGFBR3-dependent enhancement of DC-mediated tumor antigen cross presentation and $\mathrm{T}$ cell activation after chemotherapy-induced tumor cell death and antigen release. Considering the dominant immunosuppressive properties of TGF- $\beta$ and the complexities associated with measuring this cytokine in the plasma, these results suggest that sTGFBR3 levels may function as a useful predictive biomarker for tumor immunotherapy (51).

In conclusion, we have elucidated a novel mechanism for tumormediated immunoevasion that involves the downregulation of TGFBR3, a previously characterized suppressor of cancer progression. Our current data indicate that the loss of tumor-expressed TGFBR3 effectively suppressed the generation of tumor-specific immunity in both breast cancer and melanoma model systems by modulating the local TGF- $\beta$ signaling pathway in the tumor microenvironment. These findings further indicated that this alteration of local TGF- $\beta$ signaling included DC populations within the primary tumor and local TDLN tissues. As key orchestrators of the antitumor immune response, these modified DCs became capable of promoting both recruitment and activation of Tregs. Importantly, this work suggests that DC modulation is a critical component of tumor-mediated immunosuppression and that DC-specific TGF- $\beta$ signaling pathways may represent key targets for the development of tumor immunotherapies. In addition, our findings indicate that clinical trials combining a TGF- $\beta$ inhibitor with a targeted immunologic approach have great promise and that plasma sTGFBR3 may serve as an effective biomarker for selecting cancer patients for immunotherapy management.

\section{Methods}

Mice. BALB/c mice (H-2 ${ }^{\mathrm{d}}$ ) and C57BL/6 mice (H-2 $)$ (6-8 weeks of age) were obtained from Harlan Laboratories. NSG mice were purchased from Jackson Labs (stock no. 005557). Cd11 $c^{d n T G F B R 2}$ transgenic mice (H-2 $)$ (gift from R. Flavell, Yale University, New Haven, Connecticut, USA) were maintained on the C57BL/ 6 background and screened by PCR genotyping $(34,35)$.

Tissue procurement and clinical evaluation. In-transit melanoma biopsies and plasma samples were obtained as previously described from 103 and 52 patients, respectively, prior to isolated limb infusion therapy (42). Clinical responses were evaluated 3 months after ILI therapy based on RECIST1.1 criteria. Responders were defined as patients with stable disease, partial response, or complete response. Date of death was assessed based on the Social Security Death Index, and overall survival from the time of ILI therapy was calculated. Subsequent treatment regimens for patients undergoing ILI were recorded.

Cell lines. Previously described 4T1-NEO and 4T1-TGFBR3 murine mammary carcinoma cell lines $\left(\mathrm{H}-2^{\mathrm{d}}\right)(22)$ were maintained in DMEM, $10 \% \mathrm{FBS}$, and $200 \mu \mathrm{g} / \mathrm{ml} \mathrm{G} 418$. The 4T1-HER2 (H-2 ${ }^{\mathrm{d}}$ ) cell line was cultured as previously described (37). The 66CL4 murine mammary carcinoma cell line $\left(\mathrm{H}-2^{\mathrm{d}}\right)$ (gift from F. Miller, Wayne State University School of Medicine, Detroit, Michigan, USA) was cultured in DMEM, nonessential amino acids, L-glutamine, and 10\% FBS (52). The B16/F10 and B16mOVA murine melanoma cell lines $\left(\mathrm{H}-2^{\mathrm{b}}\right.$ ) (gift from T.F. Tedder and D.J. Dillilo, Duke University, Durham, North Carolina, USA) were maintained in DMEM, 10\% FBS, and $400 \mu \mathrm{g} / \mathrm{ml} \mathrm{G} 418$ (53). The D2SC/1 DC line (gift from S.-M. Kang, UCSF, San Francisco, California, USA) was maintained in IMDM, $10 \% \mathrm{FBS}$, and $20 \mu \mathrm{M} \beta$-mercaptoethanol. COS7 (ATCC no. CRL1651) and 293T (ATCC no. CRL-11268) cells were purchased from ATCC and cultured in DMEM with $10 \%$ FBS. All cell lines tested negative for mycoplasma contamination.

Reagents. The following antibodies were used for Western blot assays: pSMAD2(S465/467) (Cell Signaling, catalog no. 3101L), total SMAD2 (Cell Signaling, catalog no. 3103S), IDO (Biolegend, catalog no. 122402), CCL22 (abcam, catalog no. ab53002), PARP (Cell Signaling, catalog no. 9542), and HA (Covance, catalog no. MMS-101P). The following antibodies were used for flow cytometry (from BD Pharmingen, unless otherwise specified): B220 (catalog no. 553087), CD3 (catalog no. 551163), CD4 (catalog no. 553047), CD8 (catalog no. 553031), CD11c (catalog no. 553802), CD16/CD32 (catalog no. 553141), CD40 (catalog no. 553790), CD45 (catalog no. 550994), CD80 (catalog no. 553769), CD86 (catalog no. 553691), CCR7 (catalog no. 560766), FOXP3 (catalog no. 560408), HER2/ NEU (catalog no. 340552), PDCA-1 (Miltenyi biotec, catalog no. 130-091962), and TGFBR3 (R\&D, catalog no. FAB5034A). A PE-labeled $\mathrm{K}^{\mathrm{b}}$-SIINFEKL tetramer was purchased from Beckman Coulter for antigen-specific $\mathrm{T}$ cell flow analysis (catalog no. T10145). IHC and immunofluorescence was performed using antibodies specific to CD3 (Sigma-Aldrich, catalog no. C7930), CD8 (abcam, catalog no. ab22378), CD11b/c (ThermoScien- 
tific, catalog no. PA1-46162), FOXP3 (Biolegend, catalog no. 126402), IDO (Biolegend, catalog no. 122402), PDCA-1 (Miltenyi biotec, catalog no. 130091-962), pSMAD2 (Cell Signaling, catalog no. 3108S), and the TGFBR3 cytoplasmic domain (prepared as previously described; ref. 22). For assays requiring TGF- $\beta$ treatment, TGF- $\beta 1$ (R\&D, catalog no. $240-B-010)$ was incubated with cells for 40 minutes for pSMAD2 Western blots and for 16-18 hours for IDO and CCL22 analysis. IFN- $\gamma(200 \mathrm{U} / \mathrm{ml}$; R\&D, catalog no. 485-MI-100) was used as a positive control for IDO upregulation; IL-4 $(20 \mathrm{ng} / \mathrm{ml})$ was used as a positive control for CCL22 upregulation.

$\left[{ }^{125}\right.$ I]TGF- $\beta$ binding and crosslinking. ${ }^{[25}$ I]TGF- $\beta$ binding and crosslinking were performed as previously described (22). Briefly, cell lysate or cell CM was incubated with 100 pM [ $\left.{ }^{125} \mathrm{I}\right] \mathrm{TGF}-\beta 1$ (Perkin-Elmer, catalog no. NEX267010UC) for 3 hours at $4^{\circ} \mathrm{C}$. The [125I]TGF- $\beta$-(s)TGFBR3 complex was crosslinked with disuccinimidyl suberate and immunoprecipitated with an antibody recognizing the extracellular domain of TGFBR3 (R\&D, catalog no. AF-242-PB). SDS-PAGE was performed, and the complex was visualized by autoradiography.

Stable cell line generation. 4T1-NEO-HER2 and 4T1-TGFBR3-HER2 cell lines were generated by transfecting 4T1-NEO and 4T1-TGFBR3 cell lines, respectively, with the pLTR2-HER2/NEU-HygroR plasmid followed by selection in hygromycin $(400 \mu \mathrm{g} / \mathrm{ml})$. Cell lines were screened by flow cytometry for surface HER2/NEU expression and by anti-HA Western blot for TGFBR3 expression. B16-mOVA and B16-mOVA-TGFBR3 cell lines were generated by subcloning rat TGFBR3 from BamHI/EcoRI-digested pcDNA3.1-rTGFBR3 into the BamHI/EcoRI restriction sites of the $\mathrm{PMX}$ retroviral vector. The pMX-TGFBR3 and PMX retroviral vectors were transfected along with gag/ pol and VSVG helper plasmids into 293T cells for the generation of viral $\mathrm{CM}$, which was cocultured with the B16-mOVA cell line. These cell lines were screened by ${ }^{[25}$ I]TGF- $\beta$ binding and crosslinking. The 4 T1-sTGFBR $3_{\text {Tet }}$ cell line was engineered by amplifying the rat TGFBR3 ectodomain from a pcDNA3-sTGFBR3 plasmid using a PCR primer pair encoding the AgeI and EcoRI restriction sites, digesting the amplicon, and ligating the product inframe into a PTGIPz vector encoding a bicistronic reverse tetracycline transactivator and a puromycin resistance gene. $293 \mathrm{~T}$ cells were transfected with the final PTGIPz-sTGFBR3 ${ }_{\text {Tet }}$ vector along with the psPAX2 and pMD2.G helper plasmids, and the resulting lentiviral CM supplemented with polybrene was cocultured with a parental 4T1 cell line followed by puromycin selection. A GFP-expressing PTGIPz transfer vector was used to generate a control cell line. These cell lines were screened by sTGFBR3 Western blot and by $\left.{ }^{[125} \mathrm{I}\right] \mathrm{TGF}-\beta$ binding and crosslinking in the presence and absence of doxycycline. 66CL4-TGFBR3-KD and 66CL4-NTC cell lines were generated using the 66CL4 cell line and a TGFBR3-targeted PLKO.1-shRNA transfer vector (Sigma-Aldrich, NM_011578.3-2268s21c1) using the lentiviral protocol described above. After puromycin selection, stable cell lines were screened by TGFBR3 immunoprecipitation and Western blot analysis.

Murine bone marrow-derived DC generation. The protocol developed by Inaba et al. (54) was used to harvest murine bone marrow and generate $\mathrm{mDC}$ in the presence of GM-CSF (R\&D, catalog no. 415-ML-050) and IL-4 (R\&D, catalog no. 404-ML-050). mDCs were purified to greater than 90\% purity by flow cytometry using CD11c microbeads (Miltenyi Biotec, catalog no. 130-052-001) according to the manufacturer's recommendations. For expansion of pDCs, harvested mouse bone marrow was cultured in the presence of Flt3L (R\&D, catalog no. 427-FL-025) for 11 days before the $\mathrm{CD} 11 \mathrm{c}^{+} \mathrm{B} 220^{+} \mathrm{pDC}$ population was purified by fluorescence-activated cell sorting (FACS) (55).

Cell isolation. LNs were resected, single-cell suspensions were generated using mechanical disaggregation, and $\mathrm{pDCs}$ were purified to greater than $90 \%$ purity by flow cytometry using mPDCA-1 microbeads (Miltenyi Biotec, catalog no. 130-091-965). Spleens were resected, single-cell suspensions were generated using mechanical disaggregation, and the resulting cell suspension was spun on a Histodenz gradient (Sigma-Aldrich, catalog no. $\mathrm{D} 2158$ ) before $\mathrm{pDC}, \mathrm{mDC}$, and $\mathrm{CD}^{+} \mathrm{T}$ cell purification was performed using PDCA-1, CD11c, and CD8 microbeads, respectively (Miltenyi biotec, catalog nos. 130-091-965, 130-052-001, 130-049-401) (11).

In vivo tumor experiments. 4T1-NEO and 4T1-TGFBR3 tumor cells $\left(75 \times 10^{3}\right.$ cells) were injected into the right axillary mammary fat pad of 6- to 7-weekold female BALB/c mice, and tumor growth was measured by caliper. Tumor volumes were calculated as $\left(l \times w^{2}\right) / 2$. B16 tumor cells $\left(1 \times 10^{4}\right.$ cells $)$ were implanted subcutaneously over the shaved right anterior hemithorax of 6- to 7-week-old female C57BL/6 mice, and tumor growth was measured by caliper. Tumor, TDLN, and splenic tissues were resected between days 14 and 21 of tumor growth. A similar protocol was followed for B16 tumor implantation in $C d 11 c^{d n T G F B R 2}$ transgenic mice; however, tissues were resected up to day 30 of tumor growth. For TGF- $\beta$ inhibitor studies, 4T1-HER2 tumor cells were implanted into the right mammary fat pad of syngeneic female BALB/c mice (7 weeks of age), and growth was allowed to progress until tumors became palpable on day 11. Mice were either initiated on chow formulated with the type I TGF- $\beta$ serine/threonine kinase inhibitor SM16 (Biogen Idec) or on regular drug-free chow (Research Diets) for 3 days, before tumor-bearing mice were vaccinated and boosted 5 days later via intradermal fat pad injection either with AdHER2ki or with AdLacZ as negative control $\left(2.6 \times 10^{10}\right.$ viral particles/mouse $)(37,38,40)$. Tumor growth was monitored by caliper, and spleens were removed on day 30 after implantation for further ELISPOT analysis. For TGF- $\beta$ mAb studies, the anti-pan-TGF- $\beta$ mAb 2 G7 (Genentech) was delivered into the tumor bed of mice bearing 4T1-NEO tumors every 2-3 days after tumor implantation into the right axillary mammary fat pad $(100 \mu \mathrm{g} /$ dose $)$. For the in vivo Tet-on tumor experiments, 4 T1-sTGFBR $3_{\text {Tet }}$ tumor-bearing mice were exposed to doxycycline (up to 200 $\mu \mathrm{g} / \mathrm{ml}$ ) along with $10 \%$ sucrose in their water supply starting at various time points after tumor implantation.

In vivo tumor bioluminescence analysis. Primary 4T1 tumors were resected under sterile conditions 21 days after implantation. On day 25 , tumor burden was assessed by in vivo bioluminescence every 3 days for the next 12 days. Briefly, D-luciferin (Gold Biotechnology Inc., catalog no. LUCNA-1G) was administered to tumor-bearing mice by intraperitoneal injection at 150 $\mu \mathrm{g} / \mathrm{g}$. After 8 minutes, images were recorded and analyzed using an IVIS camera (Xenogen) as previously described (22).

cDNA filter array. cDNA filter array was performed as previously described (22). Briefly, a cDNA filter array from 10 melanoma tumors and corresponding normal tissues (Clontech) was probed with [ $\left.{ }^{32} \mathrm{P}\right]$-labeled cDNA for TGFBR3, PCR amplified, and labeled by random primed DNA using $\left[\alpha-{ }^{32} \mathrm{P}\right] \mathrm{dCTP}$.

Microarray analysis. Microarray data sets publicly available on the Oncomine Cancer Profiling Database (Oncomine 4.4, http://www.oncomine. com) were used to investigate TGFBR3 gene expression in human benign nevi and melanoma tissues as well as TGFBR3, IDO, CCL22, and FOXP3 relative gene expression in normal mammary and malignant breast cancer tissues (56). Tissue RNA preparation and microarray analysis was performed as previously described (42). Gene expression data of melanoma tumor specimens were deposited in GEO (accession no. GSE19293).

qRT-PCR. RNA was isolated from whole tissue and cells using the RNeasy Mini Kit (Qiagen, catalog no. 74106), and first strand synthesis was performed using the iScript cDNA Synthesis Kit (BioRad, catalog no. 1708891) before qRT-PCR was performed in the presence of the iQ SYBR Green SuperMix (BioRad, catalog no. 170-882) using a BioRad iCycler. See Supplemental Table 5 for primer sequences. Relative transcript levels were calculated according to the $2^{-\Delta \Delta \mathrm{Ct}}$ method, with all data normalized to Gapdh.

IHC and immunofluorescence. Tumor and TDLN tissues were resected, and paraffin-embedded tissue sections were processed according to standard protocol. Heat-induced antigen retrieval was performed in either a sodium citrate buffer or a Tris-EDTA buffer prior to blocking with $10 \%$ goat 
serum and probing for the following antigens, according to the antibody manufacturer's recommended protocol: CD3 (1:200), CD8 (1:50), FOXP3 (1:100), CCL22 (1:50), IDO $(8 \mu \mathrm{g} / \mathrm{ml})$, and TGFBR3 (1:300). HRP-conjugated secondary antibodies and the DAB substrate system was used for detection. All washes and antibody incubations were performed in TBS plus $0.1 \%$ Triton-X100. Tissue microarrays for human benign nevi and primary and metastatic melanomas (US Biomax Inc., catalog no. ME1003) and both 4 T1-sTGFBR $3_{\text {Tet }}$ and 66CL4 tumor tissues were deparaffinized, rehydrated, and blocked with Peroxidazed 1 (Biocare Medical, catalog no. PX968G) and Background Punisher (Biocare Medical, catalog no. BP974G) before incubation with the TGFBR3 cytoplasmic domain antibody, as previously described (22). This was followed by sequential treatment with an alkaline phosphatase polymer system and the Warp Red chromogen (both Biocare Medical, catalog nos. M3R533G and WR806H). FACS-sorted LNderived PDCs were spun down on poly-D-lysine-coated slides, fixed with $4 \%$ paraformaldehyde, permeabilized with $0.1 \%$ Triton-X100, and stained with the IDO-specific primary antibody.

TUNEL and PCNA staining. TUNEL analysis (TumorTACS in situ Apoptosis Detection Kit, Trevigen, catalog no. 4815-30-K) and PCNA immunostaining (Santa Cruz, catalog no. sc-9857) was performed on paraffin-embedded tumor tissue sections according to the manufacturers' instructions.

Flow cytometry. Single-cell suspensions of tumor tissues were prepared using enzymatic digestion with collagenase IV (Sigma-Aldrich, catalog no. C5138), hyaluronidase (Sigma-Aldrich, catalog no. H6254), and deoxyribonuclease (Sigma-Aldrich, catalog no. D5025) at $37^{\circ} \mathrm{C}$ for 3 hours, followed by mechanical disaggregation and red blood cell lysis. LN single-cell suspensions were prepared by mechanical disaggregation. Cells were washed with PBS containing 0.1\% BSA, Fc blocked with the anti-CD16/CD32 antibody, stained with fluorophore-labeled antibodies, and analyzed using a FACScan analyzer flow cytometer (BD).

Western blot and immunoprecipitation. Total cell lysates were harvested, boiled in sample buffer, separated by SDS-PAGE, transferred onto polyvinylidene difluoride membranes, blocked in 5\% BSA or 5\% milk in PBS with $0.1 \%$ Tween, and incubated overnight with the primary antibody of interest. For immunoprecipitation of CCL22, cell lysates were prepared at $4{ }^{\circ} \mathrm{C}$ in the presence of protease inhibitors and spun down at maximum speed, and the resulting supernatant was incubated with protein $\mathrm{A} / \mathrm{G}$ sepharose beads along with $5 \mu \mathrm{g}$ of the CCL22 polyclonal antibody at $4{ }^{\circ} \mathrm{C}$ overnight on a rotator. Sepharose beads were spun down and washed repeatedly with lysis buffer, and sample buffer was added prior to loading on a $12.5 \%$ polyacrylamide gel for separation by electrophoresis.

In-cell Western blot. Primary pDCs were plated in a round-bottomed 96-well plate, spun down, fixed in $3.7 \%$ paraformaldehyde, permeabilized with $0.1 \%$ Triton X-100, blocked by incubation in Odyssey Blocking Buffer (Licor Biosciences, catalog no. 927-40000), and incubated with the anti-IDO antibody $(8 \mu \mathrm{g} / \mathrm{ml})$ overnight at $4^{\circ} \mathrm{C}$. The plate was washed with PBS with $0.1 \%$ Tween and incubated with the secondary antibody goat anti-rat IgG IRDye 800CW (Licor Biosciences, catalog no. 926-32219). Cells were stained with Sapphire700 (Licor Biosciences, catalog no. 928-40022) at 1:1,000 dilution and DRAQ5 (eBioscience, catalog no. 65-0880-96) at 1:10,000 dilution for 1 hour at room temperature, and data were collected and normalized to background wells using an Odyssey Infrared Imaging System (Licor Biosciences).

ELISA. Capture antibody for sTGFBR3 (R\&D Systems, catalog no. AF-242-PB) was immobilized onto an E1A/R1A plate (Corning, catalog no. 3590) overnight. After washing, patient plasma samples were loaded onto the plate and incubated at room temperature for 2 hours, followed by sequential incubations with a detection antibody to sTGFBR3 (R\&D systems, catalog no. BAF-242) and Strepavidin-HRP (R\&D systems, catalog no. DY998). Finally, Fast OPD substrate (Sigma-Aldrich, catalog no. $\mathrm{P} 9187$ ) was added, $3 \mathrm{M} \mathrm{HCl}$ was applied to stop the reaction after $30 \mathrm{~min}$ - utes, and optical absorbance at $490 \mathrm{~nm}$ was recorded. CCL22 levels in the CM collected from treated bone marrow-derived DCs were analyzed using a murine CCL22 ELISA system (R\&D systems, catalog no. MCC220) according to the manufacturer's instructions.

Luciferase reporter assays. The pGL3-CCL22prom-luc luciferase reporter construct (Ccl22 promoter -722/-11, gift from K. Hieshima, Kinko University School of Medicine, Osaka, Japan) was transfected into COS7 cells and treated with IL- 4 and TGF- $\beta$ before luciferase activity was measured using a dual-luciferase reporter assay system based on the manufacturer's instructions (Promega, catalog no. E1910).

IDO enzymatic assay. The enzymatic activity of IDO was monitored using a previously established colorimetric assay (57). Briefly, treated DCs were incubated with $\mathrm{HBSS}$ and $500 \mu \mathrm{M}$ tryptophan at $37^{\circ} \mathrm{C}$ for 4 hours, and $100 \mu \mathrm{l}$ of the conditioned buffer was added to $50 \mu \mathrm{l}$ trichloroacetic acid, vortexed, and centrifuged at $9,000 \mathrm{~g}$ for 5 minutes. The resulting supernatant $(75 \mu \mathrm{l})$ was added to $75 \mu \mathrm{l}$ of $2 \%$ paradimethylaminobenzaldehyde in glacial acetic acid. The optical density of the resulting solution was measured at $492 \mathrm{~nm}$ using a Tecan monochromatic microplate reader.

$T$ cell proliferation assays. Distant LNs and TDLNs resected from BALB/c 4T1 tumor-bearing mice were dissociated into a single-cell suspension and coincubated 1:1 with allogeneic LN cells isolated from tumor-free C57BL/6 mice in a round-bottomed 96 -well plate. $\left[{ }^{3} \mathrm{H}\right]$ thymidine $(1 \mu \mathrm{Ci}$, Perkin Elmer, catalog no. NET027W) was added after 3 days of incubation, and the cells were harvested 18 hours later onto a Filtermat A (Perkin Elmer, catalog no. 1450-421) using a MACHIII automated cell harvester (Tomtec). $\left[{ }^{3} \mathrm{H}\right]$ thymidine uptake was measured using a liquid scintillation counter (Wallac). For tumor antigen-specific T cell proliferation assays, TDLN cells were pulsed with increasing concentrations of a HER2/NEU intra-cytoplasmic domain peptide mix (JPT Innovative Peptide Solutions, catalog no. PM-ERB_ICD). After 3 hours, these cells were coincubated with autologous splenocytes resected from the same HER2/NEU-expressing tumor-bearing mouse for 5-6 days before being pulsed with $\left[{ }^{3} \mathrm{H}\right]$ thymidine. Uptake was measured as described above. ConA was used as a positive control. pDC suppression assays were performed by coincubating purified TDLN-derived pDCs $(\sim 2.5 \%)$ in an allogeneic mixed lymphocyte reaction in the presence or absence of the IDO inhibitor 1-MT at $200 \mu \mathrm{M}$ (Sigma-Aldrich, catalog no. 86046). 1-MT was prepared as previously described by dissolving in $0.1 \mathrm{~N}$ $\mathrm{NaOH}$ and adjusting the $\mathrm{pH}$ to 7.5 prior to the assay $(16) .\left[{ }^{3} \mathrm{H}\right]$ thymidine was added after 3 days of incubation, and the cells were harvested 4 hours later for measurement of $\left[{ }^{3} \mathrm{H}\right]$ thymidine uptake.

IFN- $\gamma$ ELISPOT assays. Isolated LN cell cultures were coincubated with $1 \mu \mathrm{g} / \mathrm{ml}$ HER2/NEU peptide mix and murine IL-2 (BioAbChem, catalog no. 42-IL2 C) 3-10 days prior to performing an IFN- $\gamma$ ELISPOT assay according to the manufacturer's instructions (Mabtech Inc., catalog no. 3321-2AW-Plus). Influenza A HA peptide was used as an irrelevant peptide control, which generated no response. IFN- $\gamma$ ELISPOT analysis for the vaccination study was performed according to manufacturer's instructions (Mabtech Inc., catalog no. 3321-2H) using a HER2/NEU peptide mix as the stimulating antigen and HIV-irrelevant peptide mixes as negative controls (BD Biosciences). The nonspecific stimulators phorbol 12-myristate 13 -acetate and ionomycin were used as positive controls. Spot numbers are presented per $1 \times 10^{6} \mathrm{LN}$ cells.

Statistics. Data obtained from animal experiments were not assumed to be normally distributed, and nonparametric 2-tailed Mann-Whitney U tests were performed to determine statistical significance. Multiple-comparison analyses were performed using nonparametric 1-way ANOVA (KruskalWallis) followed by a Dunn's test. TGFBR3 expression levels were correlated with FOXP3 expression levels in the Riker human melanoma database (Oncomine 4.4) based on a nonparametric 2-tailed Spearman correlation calculation (56). Downregulation in TGFBR3 mRNA levels in human mela- 
noma based on DNA hybridization blot analysis was quantified by densitometric analysis (Image J) and compared using a Wilcoxon matched-pairs signed rank test (58). Correlation between TGFBR3 expression by melanoma tumor tissues based on microarray analysis and response to therapy was performed using a 2-tailed Fischer's exact test. Multivariate analysis was performed by the Biostatistical Core Resource at Duke University Medical Center to establish sTGFBR3 levels as a variable independently associated with overall survival after therapy. Overall patient survival was assessed based on data derived from the Social Security Death Index. Subsequent treatment strategies after ILI were also recorded. Association of plasma sTGFBR3 with overall survival was examined by the method of Kaplan-Meier, and statistical significance was calculated based on log-rank tests. All other comparisons were performed using 2-sided Student's $t$ tests to determine statistical significance. In all cases, a $P$ value less than 0.05 was considered significant.

Study approval. All mice were maintained in pathogen-free conditions at Duke University, and all experiments were performed in accordance with Institutional Animal Care and Use Committee protocols. Tissue and plasma samples were collected from patients with stage III in-transit melanoma, according to a protocol approved by the Duke University Medical Center Institutional Review Board (Duke IRB protocol Pro00000389). Written informed consent was obtained.

\section{Acknowledgments}

The authors thank Richard Flavell for the $C d 11 c^{d n T G F B R 2}$ transgenic mouse strain, Thomas F. Tedder and David J. Dillilo for the B16mOVA cell line, Genentech for the anti-pan-TGF- $\beta$ mAb 2G7, Kunio Hieshima for the pGL3-CCL22prom-luc reporter construct, Ursula Grohmann and Francesca Fallarino (University of Perugia, Perugia, Italy) for expertise and assistance with $\mathrm{pDC}$ isolation, Tam How and Catherine Gatza (Duke University Medical Center) for assistance with murine tumor studies, and Cheryl L. Alles for clerical assistance. The present study was funded by Department of Defense Post-doctoral Fellowship in Breast Cancer Research grant W81XWH-10-1-0850 (to B.A. Hanks), NIH grants R01CA135006 and R01-CA136786, and Komen for the Cure grants KG090154 and SAC100002 (to G.C. Blobe).

Received for publication July 11, 2012, and accepted in revised form June 13, 2013.

Address correspondence to: Gerard C. Blobe, 450 Research Drive, 354B LSRC, Box 91004, Duke University Medical Center, Durham, North Carolina 27708, USA. Phone: 919.668.1352; Fax: 919.681.6906; E-mail: gerard.blobe@duke.edu.
1. Rabinovich GA, Gabrilovich D, Sotomayor EM. Immunosuppressive strategies that are mediated by tumor cells. Ann Rev Immunol. 2007;25:267-296.

2. Gajewski T, et al. Immune resistance orchestrated by the tumor microenvironment. Immunol Rev. 2006; 213:131-145.

3. Dunn GP, Bruce AT, Ikeda H, Old LJ, Schreiber RD Cancer immunoediting: from immunosurveillance to tumor escape. Nat Immunol. 2002;3(11):991-998.

4. Zitvogel L, Tesniere A, Kroemer G. Cancer despite immunosurveillance: immunoselection and immunosubversion. Nat Rev Immunol. 2006;6(10):715-727.

5. Cochran AJ, Huang R-R, Lee J, Itakura E, Leong SPL, Essner R. Tumour-induced immune modulation of sentinel lymph nodes. Nat Rev Immunol. 2006; 6(9):659-670.

6. Enk A, Jonuleit H, Saloga J, Knop J. Dendritic cells as mediators of tumor-induced tolerance in metastatic melanoma. Int J Cancer. 1997;73(3):309-316.

7. Scarlett UK, et al. Ovarian cancer progression is controlled by phenotypic changes in dendritic cells. J Exp Med. 2012;209(3):495-506.

8. Flavell RA, Sanjabi S, Wrzesinski SH, LiconaLimon P. The polarization of immune cells in the tumour environment by TGF $\beta$. Nat Rev Immunol. 2010;10(8):554-567.

9. Banchereau J, Steinman R. Dendritic cells and the control of immunity. Nature. 1998;392(6673):245-252.

10. Belladonna ML, et al. Cutting edge: autocrine TGF- $\beta$ sustains default tolerogenesis by IDO-competent dendritic cells. J Immunol. 2008;181(8):5194-5198.

11. Pallotta MT, et al. Indoleamine 2,3-dioxygenase is a signaling protein in long-term tolerance by dendritic cells. Nat Immunol. 2011;12(9):870-878.

12. Mellor AL, Munn DH. IDO expression by dendritic cells: tolerance and tryptophan catabolism. Nat Rev Immunol. 2004;4(10):762-774.

13. Munn DH, et al. Potential regulatory function of human dendritic cells expressing indoleamine 2,3-dioxygenase. Science. 2002;297(5588):1867-1870.

14. Fallarino F, et al. The combined effects of tryptophan starvation and tryptophan catabolites downregulate $\mathrm{T}$ cell receptor $\{$ zeta\}-chain and induce a regulatory phenotype in naive $\mathrm{T}$ cells. J Immunol. 2006;176(11):6752-6761.

15. Sharma MD, et al. Plasmacytoid dendritic cells from mouse tumor-draining lymph nodes directly activate mature Tregs via indoleamine-2,3-dioxy- genase. J Clin Invest. 2007;117(9):2570-2582.

16. Munn DH, et al. Expression of indoleamine 2,3-dioxygenase by plasmacytoid dendritic cells in tumor-draining lymph nodes. J Clin Invest. 2004; 114(2):280-290.

17. Munn DH, Mellor AL. Indoleamine 2,3-dioxygenase and tumor-induced tolerance. J Clin Invest. 2007;117(5):1147-1154.

18. Curiel TJ, et al. Specific recruitment of regulatory $\mathrm{T}$ cells in ovarian carcinoma fosters immune privilege and predicts reduced survival. Nat Med. 2004;10(9):942-949.

19. Gobert M, et al. Regulatory $\mathrm{T}$ cells recruited through CCL22/CCR4 are selectively activated in lymphoid infiltrates surrounding primary breast tumors and lead to an adverse clinical outcome. Cancer Res. 2009;69(5):2000-2009.

20. Blobe GC, et al. Functional roles for the cytoplasmic domain of the type III transforming growth factor beta receptor in regulating transforming growth factor beta signaling. J Biol Chem. 2001; 276(27):24627-24637.

21. Gatza CE, Oh SY, Blobe GC. Roles for the type III TGF-beta receptor in human disease. Cell Signal. 2010;22(8):1163-1174.

22. Dong M, et al. The type III TGFbeta receptor suppresses breast cancer progression. J Clin Invest. 2007;117(1):206-217.

23. Finger E, Turley R, Dong M, How T, Fields T, Blobe G. TbetaRIII suppresses non-small cell lung cancer invasiveness and tumorigenicity. Carcinogenesis. 2008;29(3):528-535.

24. Turley R, Finger E, Hempel N, How T, Fields T, Blobe $\mathrm{G}$. The type III transforming growth factorbeta receptor as a novel tumor suppressor gene in prostate cancer. Cancer Res. 2007;67(3):1090-1098.

25 . Mythreye K, Blobe G. The type III TGF- $\beta$ receptor regulates epithelial and cancer cell migration through beta-arrestin2-mediated activation of Cdc42. Proc Natl Acad Sci U S A. 2009;106(20):8221-8226.

26. Gordon K, Dong M, Chislock E, Fields T, Blobe G. Loss of type III transforming growth factor $\beta$ receptor expression increases motility and invasiveness associated with epithelial to mesenchymal transition during pancreatic cancer progression. Carcinogenesis. 2008;29(2):252-262.

27. Andres JL, Stanley K, Cheifetz S, Massague J. Membrane-anchored and soluble forms of beta- glycan, a polymorphic proteoglycan that binds transforming growth factor-beta. J Cell Biol. 1989; 109(6 pt 1):3137-3145.

28. Lopez-Casillas F, Payne H, Andres J, Massague J. Betaglycan can act as a dual modulator of TGFbeta access to signaling receptors: mapping of ligand binding and GAG attachment sites. J Cell Biol. 1994;124(4):557-568.

29. Javelaud D, Alexaki V-I, Mauviel A. Transforming growth factor-beta in cutaneous melanoma. Pigment Cell Melanoma Res. 2008;21(2):123-132.

30. Lasfar A, Cohen-Solal KA. Resistance to transforming growth factor beta-mediated tumor suppression in melanoma: are multiple mechanisms in place? Carcinogenesis. 2010;31(10):1710-1717.

31. Arteaga C. Anti-transforming growth factor (TGF)beta antibodies inhibit breast cancer cell tumorigenicity and increase mouse spleen natural killer cell activity: implications for a possible role of tumor cell/ host TGF-beta interactions in human breast cancer progression. J Clin Invest. 1993;92(6):2569-2576.

32. Vulcano M, et al. Dendritic cells as a major source of macrophage-derived chemokine/CCL22 in vitro and in vivo. Eur J Immunol. 2001;31(3):812-822.

33. Bonecchi R, et al. Divergent effects of interleukin-4 and interferon-gamma on macrophage-derived chemokine production: an amplification circuit of polarized T helper 2 responses. Blood. 1998; 92(8):2668-2671.

34. Laouar Y, Sutterwala F, Gorelik L, Flavell R. Transforming growth factor-beta controls $\mathrm{T}$ helper type I cell development through regulatoin of natural killer cell interferon-gamma. Nat Immunol. 2005; 6(6):600-607.

35. Laouar $Y$, et al. TGF- $\beta$ signaling in dendritic cells is a prerequisite for the control of autoimmune encephalomyelitis. Proc Natl Acad Sci U S A. 2008; 105(31):10865-10870.

36. Lievens D, et al. Abrogated transforming growth factor beta receptor II (TGFbetaRII) signalling in dendritic cells promotes immune reactivity of $\mathrm{T}$ cells resulting in enhanced atherosclerosis [published online ahead of print May 21, 2012]. Eur Heart J. doi:10.1093/eurheartj/ehs106.

37. Morse MA, et al. Synergism from combined immunologic and pharmacologic inhibition of HER2 in vivo. Int J Cancer. 2010;126(12):2893-2903.

38. Suzuki E, et al. A novel small-molecule inhibitor 
of transforming growth factor beta type I receptor kinase (SM16) inhibits murine mesothelioma tumor growth in vivo and prevents tumor recurrence after surgical resection. Cancer Res. 2007;67(5):2351-2359.

39. Kim S, et al. Systemic blockade of transforming growth factor-beta signaling augments the efficacy of immunogene therapy. Cancer Res. 2008; 68(24):10247-10256.

40. Hartman ZC, et al. An adenoviral vaccine encoding full-length inactivated human Her2 exhibits potent immunogenicty and enhanced therapeutic efficacy without oncogenicity. Clin Cancer Res. 2010;16(5):1466-1477.

41. Hanks BA, Morse MA. Pharmacological inhibition of TGFbeta as a strategy to augment the antitumor immune response. Curr Opin Investig Drugs. 2010; 11(12):1342-1353.

42. Augustine CK, et al. Gene expression signatures as a guide to treatment strategies for in-transit metastatic melanoma. Mol Cancer Ther. 2010;9(4):779-790.

43. Dunn GP, Old LJ, Schreiber RD. The immunobiology of cancer immunosurveillance and immunoediting. Immunity. 2004;21(2):137-148.

44. Fallarino F, et al. Modulation of tryptophan catabolism by regulatory T cells. Nat Immunol. 2003 4(12):1206-1212.
45. Hwu P, Du MX, Lapointe R, Do M, Taylor MW, Young HA. Indoleamine 2,3-dioxygenase production by human dendritic cells results in the inhibition of $\mathrm{T}$ cell proliferation. J Immunol. 2000; 164(7):3596-3599.

46. Grohmann U, et al. CTLA-4-Ig regulates tryptophan catabolism in vivo. Nat Immunol. 2002; 3(11):1097-1101

47. Yang $P$, et al. TGF- $\beta$-miR-34a-CCL2 2 signalinginduced Treg cell recruitment promotes venous metastases of HBV-positive hepatocellular carcinoma. Cancer Cell. 2012;22(3):291-303.

48. Ramalingam R, et al. Dendritic cell-specific disruption of TGF-beta receptor II leads to altered regulatory $\mathrm{T}$ cell phenotype and spontaneous multiorgan autoimmunity. J Immunol. 2012;189(8):3878-3893.

49. Kirkbride KC, Blobe GC. Inhibiting the TGF-beta signalling pathway as a means of cancer immunotherapy. Expert Opin Biol Ther. 2003;3(2):251-261.

50. Anderton MJ, et al. Induction of heart valve lesions by small-molecule ALK5 inhibitors. Toxicol Pathol. 2011;39(6):916-924.

51. Wojtowicz-Praga S. Reversal of tumor-induced immunosuppression by TGF-beta inhibitors. Invest New Drugs. 2003;21(1):21-32.

52. Aslakson CJ, Miller FR. Selective events in the met- astatic process defined by analysis of the sequential dissemination of subpopulations of a mouse mammary tumor. Cancer Res. 1992;52(6):1399-1405

53. DiLillo DJ, Yanaba K, Tedder TF. B cells are required for optimal $\mathrm{CD}^{+}$and $\mathrm{CD}^{+} \mathrm{T}$ cell tumor immunity: therapeutic B cell depletion enhances B16 melanoma growth in mice. J Immunol. 2010;184(7):4006-4016.

54. Inaba $\mathrm{K}$, et al. Generation of large numbers of dendritic cells from mouse bone marrow cultures supplemented with granulocyte/macrophage colony-stimulating factor. J Exp Med. 1992;176(6):1693-1702.

55. Brawand P, Fitzpatrick DR, Greenfield BW, Brasel K, Maliszewski CR, De Smedt T. Murine plasmacytoid pre-dendritic cells generated from Flt3 ligandsupplemented bone marrow cultures are immature APCs. J Immunol. 2002;169(12):6711-6719.

56 . Rhodes DR, et al. ONCOMINE: a cancer microarray database and integrated data-mining platform. Neoplasia. 2004;6(1):1-6.

57. Braun D, Longman RS, Albert ML. A two-step induction of indoleamine 2,3 dioxygenase (IDO) activity during dendritic-cell maturation. Blood. 2005; 106(7):2375-2381.

58. Schneider CA, Rasband WS, Eliceiri KW. NIH Image to ImageJ: 25 years of image analysis. Nat Methods. 2012;9(7):671-675. 